OPEN ACCESS

Edited by:

Huiyu Zhou,

University of Leicester,

United Kingdom

Reviewed by:

Zheheng Jiang,

Lancaster University, United Kingdom

Jialin Lyu,

University of Leicester

United Kingdom

*Correspondence:

$\mathrm{Bin} \mathrm{Hu}$

bh@/zu.edu.cn

Zhenyu Liu

liuzhenyu@/zu.edu.cn

Specialty section:

This article was submitted to

Perception Science,

a section of the journal

Frontiers in Neuroscience

Received: 24 September 2020

Accepted: 08 March 2021

Published: 23 April 2021

Citation:

Guo W, Yang H, Liu Z, Xu Y and Hu B (2021) Deep Neural Networks for

Depression Recognition Based on $2 D$ and 3D Facial Expressions Under

Emotional Stimulus Tasks.

Front. Neurosci. 15:609760.

doi: 10.3389/fnins.2021.609760

\section{Deep Neural Networks for} Depression Recognition Based on 2D and 3D Facial Expressions Under Emotional Stimulus Tasks

\author{
Weitong Guo 1,2,3,4, Hongwu Yang ${ }^{2,4}$, Zhenyu Liu ${ }^{1,3 *}$, Yaping $\mathrm{Xu}^{1,2}$ and Bin $\mathrm{Hu}{ }^{1,3 *}$ \\ ${ }^{1}$ School of Information Science Engineering, Lanzhou University, Lanzhou, China, ${ }^{2}$ School of Educational Technology, \\ Northwest Normal University, Lanzhou, China, ${ }^{3}$ Gansu Provincial Key Laboratory of Wearable Computing, Lanzhou, China, \\ ${ }^{4}$ National and Provincial Joint Engineering Laboratory of Learning Analysis Technology in Online Education, Lanzhou, China
}

The proportion of individuals with depression has rapidly increased along with the growth of the global population. Depression has been the currently most prevalent mental health disorder. An effective depression recognition system is especially crucial for the early detection of potential depression risk. A depression-related dataset is also critical while evaluating the system for depression or potential depression risk detection. Due to the sensitive nature of clinical data, availability and scale of such datasets are scarce. To our knowledge, there are few extensively practical depression datasets for the Chinese population. In this study, we first create a large-scale dataset by asking subjects to perform five mood-elicitation tasks. After each task, subjects' audio and video are collected, including 3D information (depth information) of facial expressions via a Kinect. The constructed dataset is from a real environment, i.e., several psychiatric hospitals, and has a specific scale. Then we propose a novel approach for potential depression risk recognition based on two kinds of different deep belief network (DBN) models. One model extracts 2D appearance features from facial images collected by an optical camera, while the other model extracts 3D dynamic features from 3D facial points collected by a Kinect. The final decision result comes from the combination of the two models. Finally, we evaluate all proposed deep models on our built dataset. The experimental results demonstrate that (1) our proposed method is able to identify patients with potential depression risk; (2) the recognition performance of combined 2D and 3D features model outperforms using either 2D or 3D features model only; (3) the performance of depression recognition is higher in the positive and negative emotional stimulus, and females' recognition rate is generally higher than that for males. Meanwhile, we compare the performance with other methods on the same dataset. The experimental results show that our integrated $2 \mathrm{D}$ and $3 \mathrm{D}$ features $\mathrm{DBN}$ is more reasonable and universal than other methods, and the experimental paradigm designed for depression is reasonable and practical.

Keywords: deep belief networks, facial expression, 3D deep information, affective rating system, depression recognition 


\section{INTRODUCTION}

According to the World Health Organization (WHO), more than 350 million people of all ages suffer from depression disorder globally (Reddy, 2012). Depression (depressive disorder or clinical depression) is one of the most severe but prevalent mental disorders globally. Depression can induce severe impairments that interfere with or limit one's ability to conduct major life activities for at least 2 weeks. During at least 2 weeks, there is either a depressed mood or a loss of interest or pleasure, as well as at least four other symptoms that reflect a change in functioning, such as problems with sleep, eating, energy, concentration, self-image, or recurrent thoughts of death or suicide. Depression can occur at any age, and cases in children and adolescents have been reported ${ }^{1}$. Because of its harmfulness and recent prevalence, depression has drawn increasing attention from many related communities.

Although it is severe, depression is curable through medication, psychotherapy, and other clinical methods (National Collaborating Centre for Mental Health, 2010). The earlier that treatment can begin, the more effective it is. Thus, the early detection of depression is critical to controlling it at an initial stage and reducing the social and economic burden related to this disease. Traditional diagnosis approaches for depression are mostly based on patients self-reporting in clinic interviews, behaviors reported by relatives or friends, and questionnaires, such as the Patient Health Questionnaire (PHQ-9) (Kroenke and Spitzer, 2002) and the Beck Depression Inventory (BDI-II) (McPherson and Martin, 2010). However, all of them utilize subjective ratings, and their results tend to be inconsistent at different times or in different environments. During the diagnosis, several clinical experts must be involved to obtain a relatively objective assessment. As the number of depressed patients increases, early-stage diagnosis and reassessments for tracking treatment effects are often limited and time consuming. Therefore, machine learning-based automatic potential depression risk detection or depression recognition is expected to facilitate objective and fast diagnosis to ensure excellent clinical care quality and fundamentally reduce potential harm in real life.

Under the influence of depression, behavior disorder-based signals for depression recognition are increasingly extensive, such as voices (Ooi et al., 2013; Yang et al., 2013; Nicholas et al., 2015; Jiang et al., 2017), facial expressions (Schwartz et al., 1976; Babette et al., 2005), gestures (Alghowinem et al., 2018), gaits (Michalak et al., 2009; Demakakos et al., 2015), and eye movements (Winograd-Gurvich et al., 2006; Alghowinem et al., 2013; Carvalho et al., 2015). This work focuses on using facial expressions to recognize patients with potential depression risk. The research on depression based on facial expression essentially utilize video or images (Gupta et al., 2014; Alghowinem, 2015; Pampouchidou et al., 2015, 2016a; Bhatia et al., 2017). To be more precise, the interests are localized on images, facial landmark points (Stratou et al., 2014; Morency et al., 2015; Nasir et al., 2016; Pampouchidou et al., 2016b), and/or facial

$\overline{{ }^{1} \text { Available at: http://medlineplus.gov/depress.html. }}$ action units (AUs) (Cohn et al., 2009; McIntyre et al., 2009; Williamson et al., 2014). However, the methods that adopt image analysis (the essence of the video-based method are still images analysis where videos are converted into images) are affected by environmental factors and instrument parameters, such as illumination, angle, skin color, and resolution power. If these factors are not addressed appropriately, the recognition performance will be affected. Several researchers (Gong et al., 2009; Zhao et al., 2010) proposed using in-depth information captured from 3D sensors, which is relatively illumination, angle, and skin color invariant. However, 3D points of information can lose the texture features of facial expression. Therefore, the fusion of $2 \mathrm{D}$ with $3 \mathrm{D}$ data can make up for each other to address these issues.

Depression recognition typically comprises two steps: feature extraction and recognition (depression or not/ depression severity). The quality of feature extraction directly affects the result of recognition. Conventional feature extraction methods for depression facial expression utilize geometric features, appearance features, and dynamics. These methods extract the displacement of facial edges, corners, coordinates (McIntyre et al., 2010; Bhatia et al., 2017), mean squared distance of all mouth landmarks to the mouth centroid (Gupta et al., 2014), and displacement from the mid-horizontal axis to depict the changes and intensity of basic expressions (Bhatia, 2016). The local binary pattern (LBP), LBP-TOP (Joshi et al., 2012), local Gabor binary pattern (LGBP-TOP) (Sidorov and Minker, 2014), local curvelet binary pattern (LCBP-TOP) (Pampouchidou et al., 2015), and LPQ from three orthogonal planes (LPQTOP) (Wen et al., 2015) extracted describe the texture changes in the facial region. Histogram of optical flow (Gupta et al., 2014), motion history histogram (MHH) (Meng et al., 2013), and space-time interest points (STIPs) (He et al., 2015) are extracted to describe the facial motion. These results indicated that depressed people display a lower performance when responding to positive and negative emotional content. Nevertheless, all those mentioned approaches are hand-crafted feature descriptors designed based on tremendous professional knowledge, and image processing is also complicated for hand-crafted features. However, our cognition of depression remains insufficient. Such features probably yield segmented representations of facial expressions and are insufficiently discriminative. Simultaneously, the dynamics are extracted from a video, which involves the effect of the environmental factors mentioned above. On the other hand, the time window is used to extract motion features (Pampouchidou et al., 2016a; He et al., 2018). The reported window lengths are 60 frames, 20 frames, 5 frames, or even 300 frames. However, the optimal window length cannot be determined because there are significant variations over time in the facial expression according to the particular person and experimental device.

In recent years, deep learning techniques have prevailed in audio- and video-based applications, especially in visual information processing (Girshick et al., 2014). The purpose of this study is to identify the patients at risk of depression. The selected subjects are outpatients, and the evaluated depression degree is moderate. Many samples with depression risk and 
the normal control group had no noticeable expression changes in some stimulation tasks. Therefore, we chose the generative model deep belief network (DBN). The DBN-based deep learning method can hierarchically learn good representation from original data; thus, the learned facial features should be more discriminative than hand-crafted features for depression recognition. Long short-term memory (LSTM) is an effective and scalable model for learning problems related to sequential data and can capture long-term temporal dependencies. Facial expression is a dynamic process of continuous change, and it is a time-series signal on a timeline. Then facial expression motion is captured by LSTM used on the entire timeline.

The availability of clinical data is critical for the evaluation of methods for depression recognition. Because of the sensitivity of clinical data and privacy reasons, datasets for depression research are neither extensive nor free. It is why most research groups resort to generating their datasets. The current datasets are as follows: Pittsburgh, BlackDog, DAIC-WOZ, AVEC, ORI, ORYGEN, CHI-MEI, and EMORY, but only three of which are available. AVEC is the only fully public dataset available for free download, DAIC-WOZ is partly available, while Pittsburgh is also available, but not accessible now. The rest depressionrelated datasets are proprietary, and the corresponding research results are few. The securable datasets above provide the thirdparties visual and audio features. Only AVEC discloses complete video recordings. However, these datasets are collected from non-Chinese subjects, which differ from Chinese subjects in terms of emotional expressiveness due to different cultural backgrounds. Thus, we used a structured experimental paradigm to construct a depression database specifically for Chinese subjects in conjunction with relevant psychiatric hospitals. To the best of our knowledge, the database we have established is the only database with complete data, a reasonable structure, and the largest number of subjects in China. Our dataset includes complete video recordings from typical webcam and microphone, and 3D 1,347 facial points scan from deep camera Kinect (Leyvand et al., 2011). Not only does a Kinect detect the human face, but it also provides real-time access to over 1,000 facial points in the $3 \mathrm{D}$ space irrespective of the color of the skin or the surrounding environment, illumination, or distance from the camera.

This paper builds on our previous work (Guo et al., 2019) by adding $2 \mathrm{D}$ static image information and $3 \mathrm{D}$ facial point motion information to identify depression, and it is a further improvement and summary of the original work. We build two different deep networks, respectively, one of which extracts static appearance feature using 2D images based on DBN, and the other learns the facial motion via 3D facial landmark points and facial AUs using DBN-LSTM. The two kinds of deep networks are then integrated by joint fine-tuning, which can further improve the overall performance. Therefore, our main contributions in this paper can be summarized as follows:

1. We designed a reasonable and effective experimental paradigm, collected diversified data and three kinds of samples (normal population, outpatients, and inpatients) combined with specialized hospitals, and constructed a large-scale dataset for depression analysis.
2. The two deep networks proposed can extract appearance features from $2 \mathrm{D}$ images and motion features from 3D facial landmark points. The integrated networks can achieve the fusion of static and dynamic features, which can improve recognition performance.

3. We have proved qualitatively and quantitatively that depressive prone groups show significant differences from healthy groups under positive and negative stimuli.

The following section briefly describes the related works on depression recognition based on facial expression. In section 3 , we introduce the proposed depression recognition network structure. Dataset creation, experimental setting, results, and analysis are reported in section 4. Finally, some discussions and future works are provided in section 5 .

\section{RELATED WORK}

\subsection{Depression Recognition Based on Machine Learning}

Machine learning tools for depression detection have access to the same streams of information that a clinician utilizes for diagnosis. For example, the variation of facial expression, gesture, voice, and language should occur in communication modality. Reduced emotional expression variability is commonly found in depression and connected with deficits in expression positive and negative emotion (Rottenberg et al., 2005). In the following, we briefly summarize some excellent research results on identifying depression from visual cues.

Wang et al. (2008) extracted geometric features from 28 regions formed by $582 \mathrm{D}$ facial landmarks to characterize facial expression changes. Probabilistic classifiers were employed to propagate the probabilities frame by frame and create a probabilistic facial expression profile. The results indicate that depressed patients exhibit different trends of facial expressions than healthy controls. Meng et al. (2013) employed motion history histogram $(\mathrm{MHH})$ to capture motion information of facial expression. Local binary patterns (LBP) and edge orientation histogram $(\mathrm{EOH})$ features were then extracted, and partial least square (PLS) was finally applied for prediction. These features were extracted from images. Nasir et al. (2016) employed perceptually motivated distance and area features obtained from facial landmarks to detect depression. The window-based representation of features was used to capture large-scale temporal contexts results. Anis et al. (2018) developed an interpretable method of measuring depression severity. Barycentric coordinates of facial landmarks and rotation matrix of $3 \mathrm{D}$ head motion were used to extract kinematic features, and a multi-class SVM was used to classify the depression severity.

The methods mentioned above are based on traditional machine learning methods to extract hand-crafted facial expression feature descriptors for depression analysis. Some studies have also utilized deep learning to extract high-level semantic features of facial expressions from raw video recordings for automatic depression detection. Jan et al. (2018) utilized convolutional neural networks (CNN) to extract many different 
visual primitive features from the facial expression frames, while feature dynamic history histogram (FDHH) was employed to capture the temporal movement on the features. Zhou et al. (2020) presented a DCNN regression model with a GAP layer for depression severity recognition from facial images. Different face regions were modeled, and then these models were combined to improve the overall recognition performance. The results indicated that the salient regions for patients with different depression levels were usually around the eyes and forehead. Melo et al. (2019) used two 3D CNNs to model the spatiotemporal dependencies in global and local facial regions captured in a video, and then combine the global and local 3D CNNs to improve the performance. The CNN-based method mentioned above requires a large amount of data to train the model. Once the amount of data is small, it is easy to fall into overfitting. By comparing the data volume of existing studies with our method, we found that the state-of-the-art research used about 4,350 min of video-based publicly available datasets, while the amount of video data we used was only about 2,080 min. Existing studies have shown that the generation model has a better classification effect than the discriminant model in low samples (Ng and Jordan, 2002). So, we finally choose to use the DBN model.

\subsection{DBN}

The DBN (Hinton et al., 2006) is a generative model that uses multiple layers of feature-detecting neurons. It can learn hierarchical representation from raw input data and can be effectively built by stacking a restricted Boltzmann machine (RBM) (Fischer and Igel, 2012) layer-by-layer and greedily training it. In our study, the Gaussian-Bernoulli RBM is adopted to use real-valued visible units to train the first layer of the DBN; binary hidden units are used for training the higher layers. For a Gaussian-Bernoulli RBM, the energy function of a joint configuration is given as Equation (1).

$\mathrm{E}(\mathrm{V}, \mathrm{H})=\frac{1}{2 \sigma^{2}} \sum_{i=1}^{m} \frac{\left(v_{i}-a_{i}\right)^{2}}{2}-\frac{1}{\sigma^{2}}\left(\sum_{i=1}^{m} \sum_{j=1}^{n} w_{i j} v_{i} h_{j}+\sum_{j=1}^{m} b_{j} h_{j}\right)$

where $a \in R^{D}$ and $b \in R$ are the biases for visible and hidden units, respectively. $w_{i j} \in R$ is the weight between the visible unit $i$ and the hidden unit $j$, while $m$ and $n$ are the numbers of visible and hidden units, respectively. $\sigma$ is a hyper-parameter. As there are no connections between units in the same layer, the conditional probability distributions are given by Equations (2) and (3).

$$
\begin{gathered}
\mathrm{P}\left(h_{j}=1 \mid v\right)=\operatorname{sigmoid}\left(\frac{1}{\sigma^{2}}\left(\sum_{i=1}^{m} w_{i j} h_{j}+b_{j}\right)\right) \\
\mathrm{P}\left(v_{i} \mid h\right)=\mathcal{N}\left(a_{i}+\sum_{j=1}^{n} w_{i j} h_{j}, \sigma^{2}\right)
\end{gathered}
$$

where $\mathcal{N}(\mu, v)$ is a Gaussian function with mean $\mu$ and variance $v$. $(w, a, b)$ are the parameters of the RBM and are learned using contrastive divergence. The generated features are the posteriors of the hidden units in the case of given visible units. Finally, the top output values are classified using sigmoid activation and the stochastic gradient descent method is used to train the deep networks.

\subsection{LSTM}

The LSTM block has a memory cell that stores information with long-term dependencies. We use an LSTM with a conventional structure, as shown in Figure 1.

In Figure 1, $x^{(t)}$ is the input data in time step $t$ (the current frame), $h^{(t-1)}$ is the hidden unit in time step $t-1$ (the previous

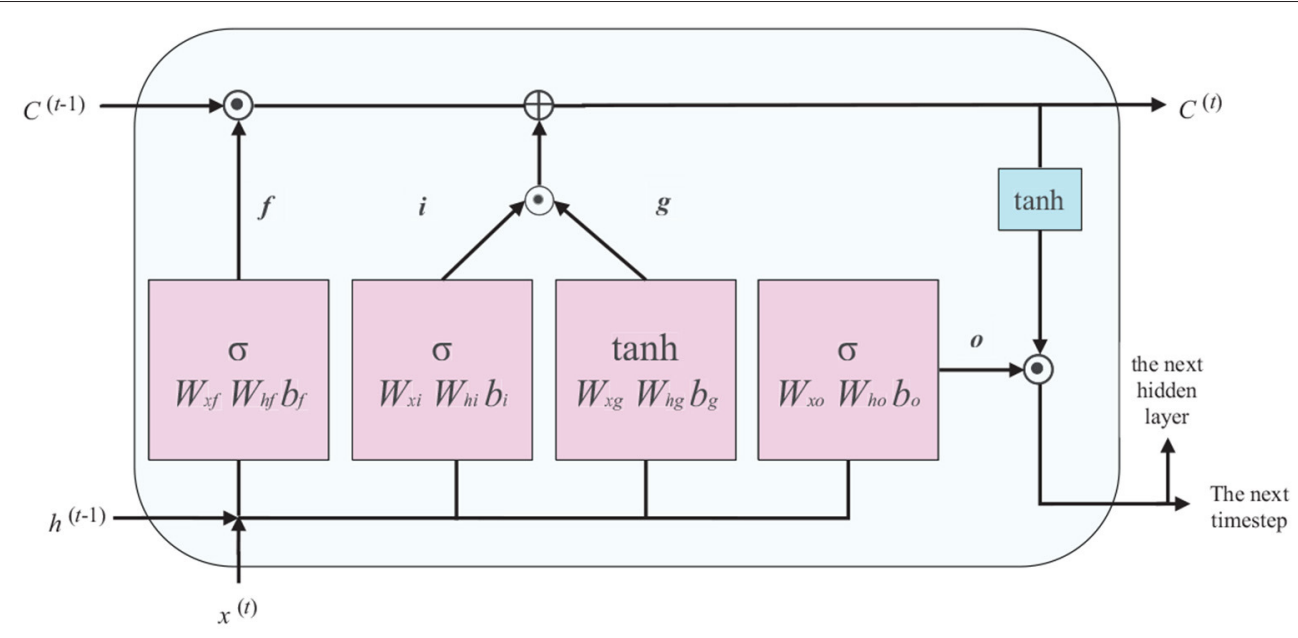

FIGURE 1 | Deployment structure of long short-term memory (LSTM) unit. 
frame), and $C^{(t-1)}$ represents the cell status of the previous time step, which is modified to obtain the cell status of the current timestep $C^{(t)}$. The flow of information in the LSTM is controlled by the computing unit described in Figure 1, namely the forget, input, and output gates. The specific process is described by the following equations:

forget gate $f_{t}$ : control the retention and removal of features.

$$
f_{t}=\operatorname{sigmoid}\left(W_{x f} x^{(t)}+W_{h f} h^{(t-1)}+b_{f}\right)
$$

input gate $i_{t}$ : update cell status with input node $g_{t}$.

$$
\begin{gathered}
i_{t}=\operatorname{sigmoid}\left(W_{x i} x^{(t)}+W_{h i} h^{(t-1)}+b_{i}\right) \\
g_{t}=\tanh \left(W_{x g} x^{(t)}+W_{h g} h^{(t-1)}+b_{g}\right) \\
C_{t}=\left(C^{(t+1)} \odot f_{i}\right) \oplus\left(i_{t} \odot g_{t}\right)
\end{gathered}
$$

output gate $O_{t}$ : update the value of a hidden unit.

$$
\begin{gathered}
O_{t}=\operatorname{sigmoid}\left(W_{x o} x^{(t)}+W_{h o} h^{(t-1)}+b_{o}\right) \\
h_{t}=O_{t} \odot \tanh \left(C^{(t)}\right)
\end{gathered}
$$

where the weight matrix subscripts have the obvious meaning. For example, $W_{h f}$ is the hidden-forget gate matrix, and $W_{x i}$ is the input-input gate matrix. The bias terms $b$, the subscripts of $f, i, g$, and $o$, denote the corresponding door's bias.

\subsection{Problem Setup}

We find that the various kinds of effective methods proposed are based on $2 \mathrm{D}$ images (video is split into images) and 2D landmark point data (extracted from 2D images) by a survey of the current research on depression based on visual cues. The main limitations of 2D image-based analysis are problems associated with large variations in pose, illumination, angle, skin color, and resolution power. Nevertheless, depth information captured from 3D sensors is relatively posed and illumination invariant. Inspired by the idea of Aly et al. (2016), the fusion of $2 \mathrm{D}$ with $3 \mathrm{D}$ data can address these issues and cover the shortage of each other that 3D landmark points miss texture feature. Each expression can be decomposed into a set of semantic AUs, which exhibit in different facial areas and at different times with different intensities. Therefore, the dataset we build contains both $2 \mathrm{D}$ video and 3D landmark points and AUs information. In the paper, we propose a novel approach for depressive prone patients recognition based on two kinds of different DBN models combination, one of which extracts $2 \mathrm{D}$ appearance features from facial images collected by optical cameras, the other learns the facial motion from 3D facial points and facial AUs collected by a Kinect. The final decision result comes from the combination of the two networks. Finally, we evaluate all proposed deep models in our built dataset and analyze three aspects: gender, stimulus task, and affective valence.

\section{PROPOSED APPROACH}

\subsection{The Framework of Deep Neural Networks-based Depression Recognition}

We utilize the DBN and LSTM to potential depression risk recognition. We build two different deep networks: $2 \mathrm{D}$ static appearance deep network (2D-SADN), which is used to extract the static appearance features from images based on DBN. In other words, the network only focused on the analysis of appearance from static facial pictures in which a single image was used as input to the network and the network structure did not encode temporal information. 3D dynamic geometry deep network (3D-DGDN) based on combined of DBNs and LSTM, which capture the dynamic geometry features of $3 \mathrm{D}$ facial landmark points and AUs from Kinect. Expressions are inherently dynamic events consisting of onset, apex, and offset phases (Liu et al., 2014). Therefore, in the second network, we took the facial contour map composed of facial landmark points as input and used the position offset of the threedimensional coordinate value on the time axis to obtain motion information. Finally, the two networks are integrated to improve the recognition performance. The overview of the proposed approach is shown in Figure 2.

\subsubsection{The Structure of DBN Model}

The designed basic DBN network is composed of four RBMs, as shown in Figure 3. First, Gibbs sampling and contrastive divergence are adopted to train RBM to maximize $\mathbb{E}_{V \sim p_{\text {data }}} \log p(v)$. The RBM parameter defines the parameters of the first layer of the DBN. Then, the second RBM is trained to approximately maximize $\mathbb{E}_{V \sim p_{\text {data }}} \mathbb{E}_{h^{(1)} \sim p^{(1)}\left(h^{(1)} \mid v\right)} \log p^{(2)}\left(h^{(1)}\right)$, where $p^{(1)}$ is the probability distribution represented by the first $\mathrm{RBM}$, and $p^{(2)}$ is the probability distribution represented by the second RBM. That is, the second RBM is trained to simulate the distribution defined by the hidden unit sampling of the first $\mathrm{RBM}$, which is driven by the input data. This process is repeated four times to add four hidden layers to the $\mathrm{DBN}$, and each new RBM models the samples of the previous RBM. Each RBM defines another layer of DBN. Top-down fine-tuning is used to generate weights to guide the determination of the DBN model. At the top two levels, the weights are linked together so that the output of the lower level will provide a correlation to the top level, which will then link it to its associative memory. DBN can adjust the discriminant performance by using the labeled data and BP algorithm.

\subsubsection{Learning the Static Appearance Deep Network}

In the $2 \mathrm{D}-\mathrm{SADN}$, we train a $\mathrm{DBN}$ as shown above with four layers by oneself with three channels, and then average the predicted values of each channel. The result is the final predicted value, as shown in Figure 4.

\subsubsection{Learning the Dynamic Geometry Deep Network} In the $3 \mathrm{D}$ dynamic geometry deep network (3D-DGDN), we build four different DBN models based on our designed basic DBN network, as shown in Figure 5. Figure 5A is a four-hidden layer DBN with facial points, named $4 \mathrm{DBN}$; Figures 5B,C shows 


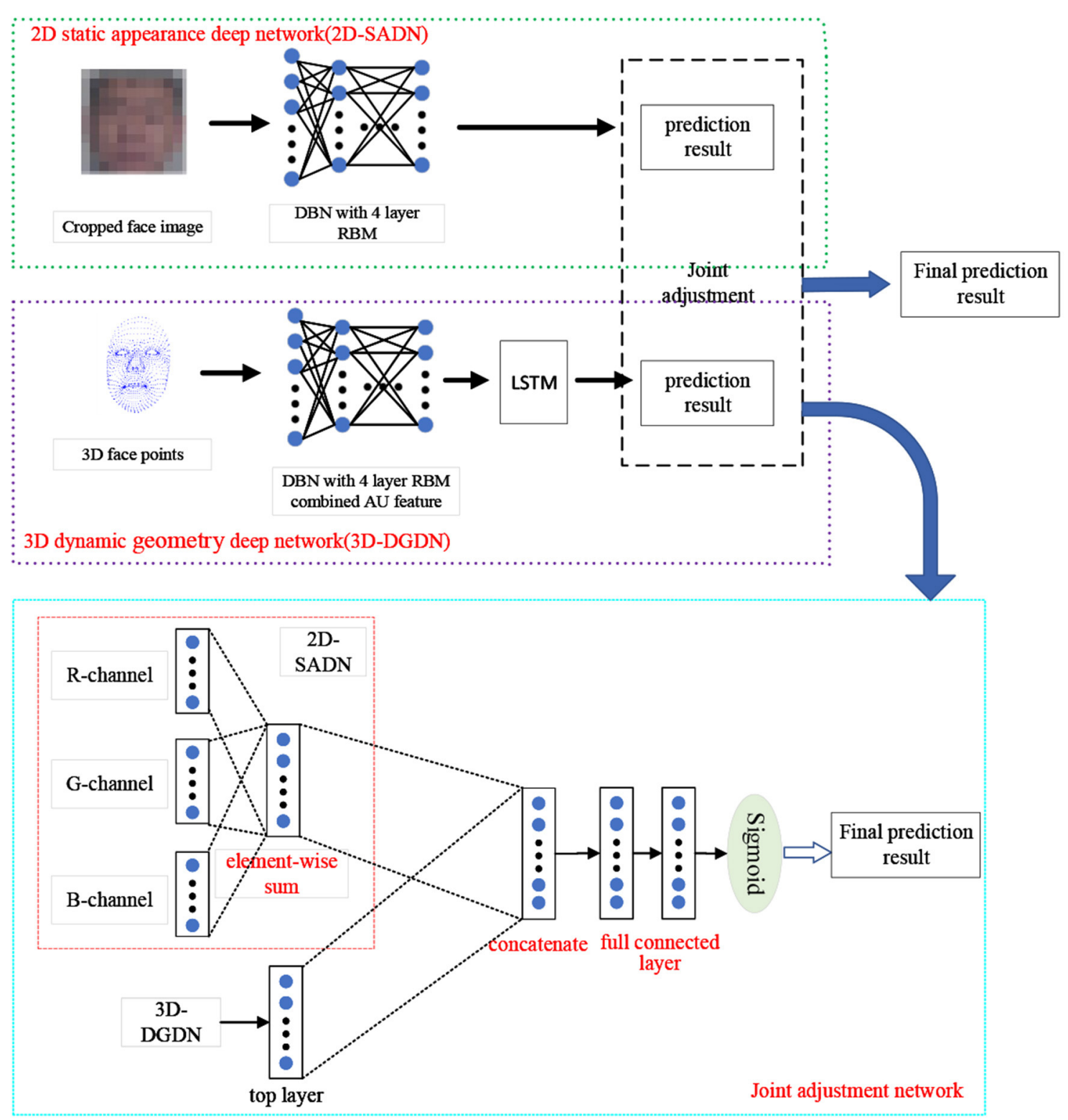

FIGURE 2 | The framework of proposed approach.

four hidden layer DBNs with facial points and AU, named AU4DBN and 4DBN-AU. Figure 5D shows a four hidden layer DBNs with facial points and AU followed by a LSTM, named AU-4DBN-LSTM. In the meantime, we build a five-hidden-layer DBN using facial points as the input and find that the accuracy rate of the four-hidden layer-DBN is almost the highest in all of the stimulus tasks, therefore a four-hidden-layer network is used as the basic DBN structure and AU-4DBN-LSTM stands for 3D-DGDN. The details are as follows.

- $4 D B N$ is a four-hidden layer DBN only using facial points as the input, as shown Figure 5A.

- $4 D B N-A U$ is a four-hidden layer DBN based on $4 \mathrm{DBN}$ that uses AU and facial points as the input, as shown Figure 5B.
- $A U-4 D B N$ is a four-hidden-layer DBN with $\mathrm{AU}$ added at the penultimate layer, which is used as the input of stacking an extra RBM on the top, as shown in Figure 5C.

- $A U-4 D B N-L S T M$ is based on the AU-4DBN model to add the LSTM. That is, the output of the RBM on the top of AU-4DBN is used as the input to the first layer of the LSTM (Greff et al., 2016), which has two layers, as shown in Figure 5D.

\subsubsection{Joint Fine-turning Method}

The strategy of adding the corresponding position elements was used to connect the activation values of the top hidden layers of three channels to obtain the feature vector in the 2D-SADN. We then concatenated the feature vector and the activation values of 


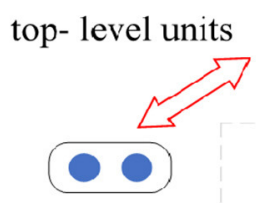

2 label units
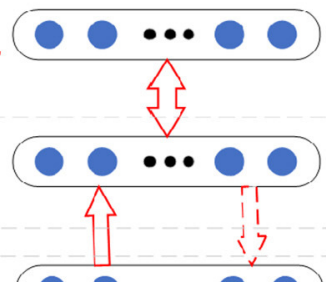

$\frac{1}{7}$

hidden4

hidden 3

detection weights

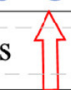

$\bullet \bullet$

$-11$ generative weights

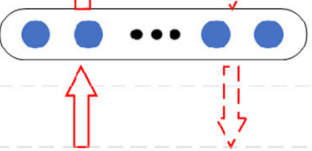

hidden 2

hidden 1

visible

\section{associative memory}

RBM

\section{RBM}

\section{RBM}

RBM

FIGURE 3 | The structure of designed deep belief network (DBN) model.

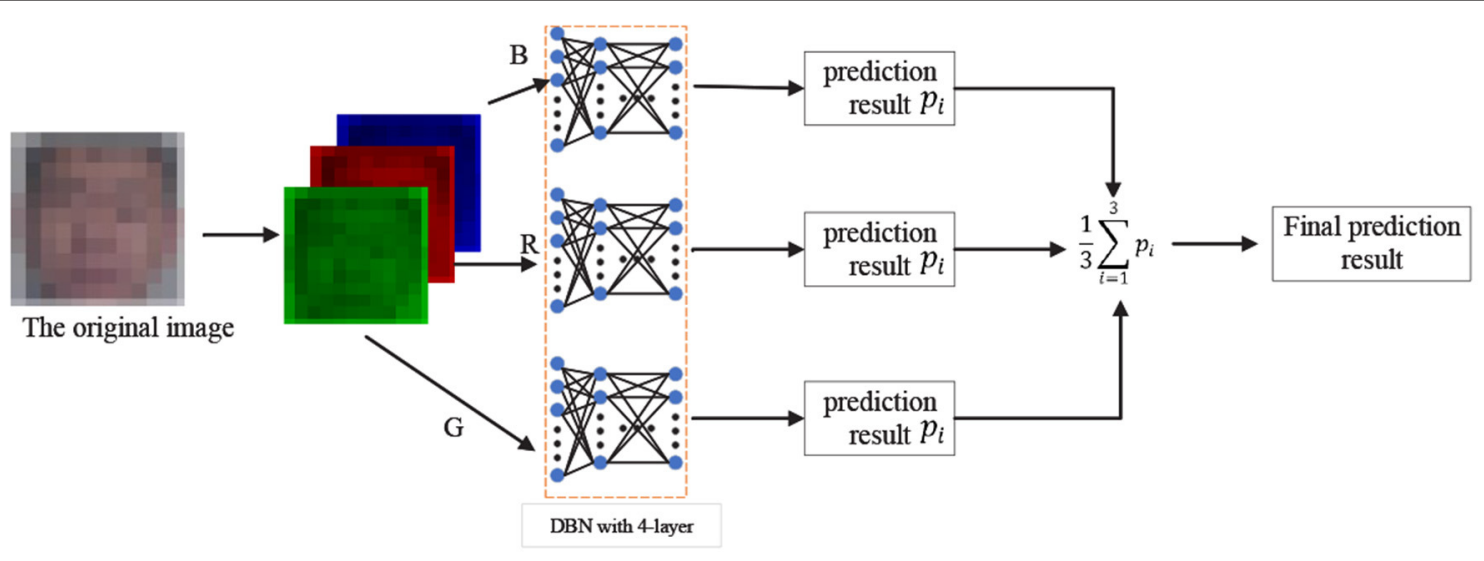

FIGURE 4 | 2D static appearance deep network.

the top hidden layers of 3D-DGDN. Finally, the concatenated values are used for inputs to a fully connected network with a sigmoid activation, as shown in Figure 2. Consequently, we integrate the network using a linear weighted sum of the loss function based on Jung et al. (2015), defined as follows:

$$
L_{\text {fusion }}=\lambda_{1} L_{2 D-S A D N}+\lambda_{2} L_{3 D-D G D N}+\lambda_{3} L_{2 D-3 D}
$$

where $L$ is cross entropy loss function, and $L_{2 D-S A D N}, L_{3 D-D G D N}$, and $L_{2 D-3 D}$ are computed by $2 \mathrm{D}-\mathrm{SADN}, 3 \mathrm{D}-\mathrm{DGDN}$, and the both, respectively. $\lambda_{1}, \lambda_{2}$, and $\lambda_{3}$ are turning parameters. In order to fully utilize the capabilities of the two models, we set the value of $\lambda_{1}, \lambda_{2}$, and $\lambda_{3}$ to 1,1 , and 0.1 , respectively. Cross entropy loss function is defined as follows:

$$
\begin{aligned}
L_{2 D-S A D N} & =-\sum_{i=1}^{n} y^{(i)} \log \hat{y}_{2 D-S A D N}^{(i)} \\
& +\left(1-y^{(i)}\right) \log \left(1-\hat{y}_{2 D-S A D N}^{(i)}\right) \\
L_{3 D-D G D N} & =-\sum_{i=1}^{n} y^{(i)} \log \widehat{y}_{3 D-D G D N}^{(i)} \\
& +\left(1-y^{(i)}\right) \log \left(1-\hat{y}_{3 D-D G D N}^{(i)}\right) \\
L_{2 D-3 D} & =-\sum_{i=1}^{n} y^{(i)} \log \hat{y}_{2 D-3 D}^{(i)}+\left(1-y^{(i)}\right) \log \left(1-\hat{y}_{2 D-3 D}^{(i)}\right)
\end{aligned}
$$




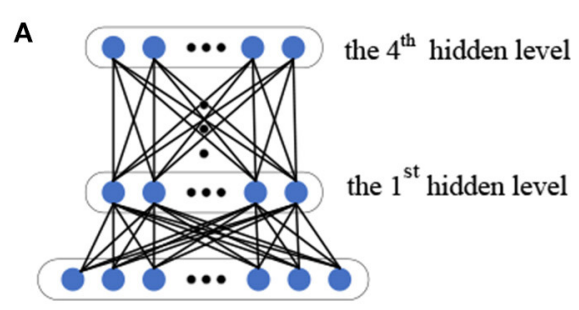

facial point input

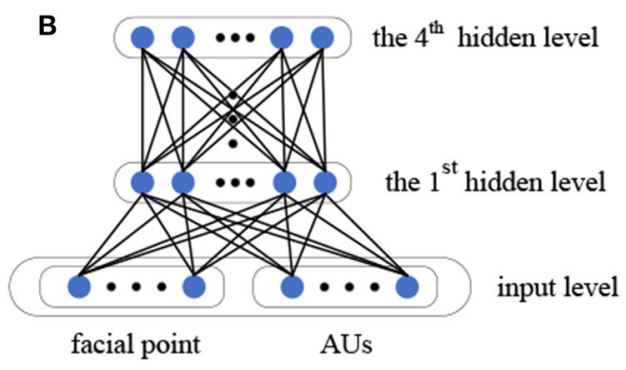

D

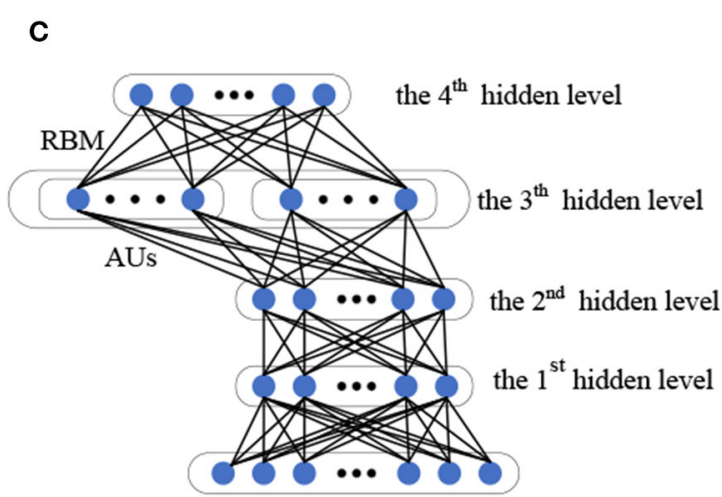

facial point input

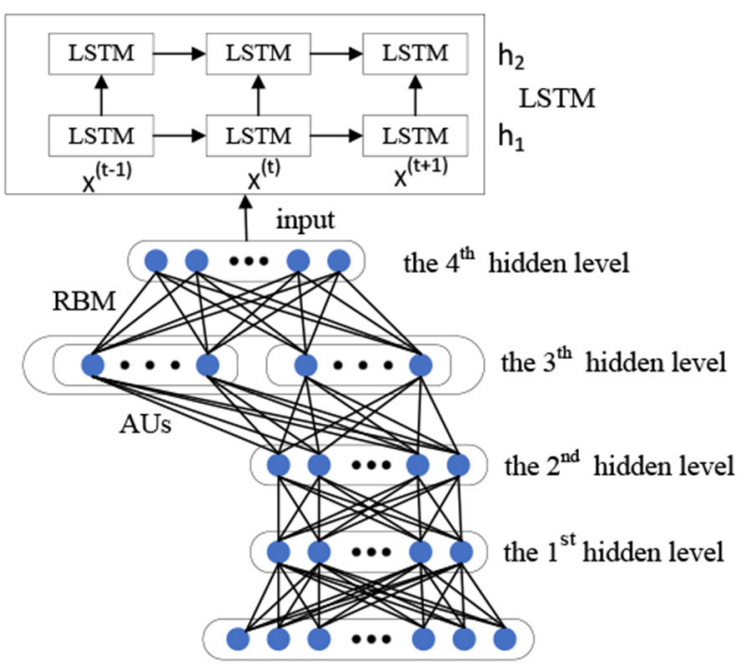

facial point input

FIGURE 5 | Framework of four different deep belief network (DBN) models: (A) 4DBN, (B) 4DBN-AU, (C) AU-4DBN, (D) AU-4DBN-LSTM.

where $n$ is the number of samples and $y^{(i)}$ is the ground-truth label of the $i$ th sample. $\hat{y}_{2 D-S A D N}^{(i)}, \hat{y}_{3 D-D G D N}^{(i)}$, and $\hat{y}_{2 D-3 D}^{(i)}$ are the $i$ th output value of sigmoid activation of $2 \mathrm{D}-\mathrm{SADN}, 3 \mathrm{D}-\mathrm{DGDN}$, and the integrated network, respectively. $\hat{y}_{2 D-3 D}^{(i)}$ is computed by logit values of network $2 \mathrm{D}-\mathrm{SADN}$ and $3 \mathrm{D}-\mathrm{DGDN}$ as follows:

$$
\hat{y}_{2 D-3 D}^{(i)}=\sigma\left(l_{2 D-S A D N}^{(i)}+l_{3 D-D G D N}^{(i)}\right)
$$

where $l_{2 D-S A D N}^{(i)}$ and $l_{3 D-D G D N}^{(i)}$ are the $i$ th logit values of network 2D-SADN and 3D-DGDN, respectively. $\sigma(\bullet)$ is a sigmoid activation function.

$$
l_{k}^{(i)}=\log \left(\frac{x_{i}}{1-x_{i}}\right)_{k} \quad \forall x_{i} \in(0,1)
$$

where $k$ means network 2D-SADN and 3D-DGDN, and $x_{i}$ is the $i$ th output value of sigmoid of network $k$. The final prediction is the index with the maximum value from the output of sigmoid of the integrated network as follows:

$$
\hat{P}=\underset{i}{\operatorname{argmax}} \hat{y}_{2 D-3 D}^{(i)}
$$

The paper uses 10-fold cross-validation to evaluate experiments for excluding the differences caused by individuality and over-fitting. Note that $80 \%$ of samples from the total participants are used for training, $10 \%$ for validation, and the rest of $10 \%$ for testing. Each fold includes the data from 42 participants for training, 5 participants for validation, and 5 participants for testing. We use accuracy to evaluate the proposed model performance. Accuracy is computed by the confusion matrix consisting of the number of true positives $(T P)$, true negatives $(T N)$, false positives $(F P)$, and false negative $(F N)$, defined as follows:

$$
\text { accuracy }=\frac{T P+T N}{T P+T N+F P+F N}
$$

where $T P$ is the number of depression samples predicted to be depressed, $T N$ is the number of healthy samples predicted to be healthy, FP is the number of healthy samples predicted to be depression, and $F N$ is the number of depressed samples predicted to be healthy. 


\section{EXPERIMENTS}

\subsection{Depression Data Collection}

To effectively obtain depression data, we cooperated with Tianshui Third People's Hospital in Gansu province to collect data. Data collection was accomplished in an isolated, quiet, and soundproof room without electromagnetic interference. Two people were present in the room at the same time: one was the clinician controlling the data collection process, and the other was the participant. The clinician operated one of the two computers and played all the stimulus tasks (film clips, voice responses, text reading, and picture description) sequentially. The stimulus materials were displayed to the participant on the second computer. Participants must evaluate their emotional state before and after completing each stimulus task. Each stimulus task was divided into positive, neutral, and negative stimuli. In order to prevent the stimulus of the previous emotional valence from affecting the next emotional valence stimulus, there is a 1 -min break at the end of each material. Moreover, the order of valence stimulation presented to each subject was also different. Audio and video information on the participant was recorded by a webcam, a Kinect camera, and a microphone.

\subsubsection{Participants}

Every participant is rated by psychiatrists through interviews and questionnaires. The set of questionnaires required to be filled included the International Neuropsychiatric Interview (MINI) and the Patient Health Questionnaire-9 (PHQ-9). PHQ-9 was the main grouping criteria (health control: $<5$, patient: $\geq 5$ ). PHQ-9 scores are treated as the label. In this experiment, the outpatient sample set included data from 52 males and 52 females; meanwhile, the control group also included data from 52 males and 52 females. Participants were excluded from the health group if they received a Beck depression inventory (BDI) score $>5$. The demographic characteristics of all participants are shown in Table 1. All participants provided informed consent.

\subsubsection{Paradigm Design of Depression Experiments}

Depressed individuals have negative self-schema in cognitive processing related to attention control disorder of emotional interference. The phenomena related to emotion include subjective experience, facial expression behavior, individual

TABLE 1 | Demographic characteristic of the out-patients and control group: mean and (standard deviation).

\begin{tabular}{llccccc}
\hline Gender & Category Number & Age & Education & PHQ-9 & BDI \\
\hline Male & $\begin{array}{l}\text { Control } \\
\text { group } \\
\text { Out- } \\
\text { patients }\end{array}$ & 52 & $39(10.8)$ & $11.8(2.5)$ & $0.8(2.0)$ & $6.4(6.4)$ \\
\hline Female & $\begin{array}{l}\text { Control } \\
\text { group } \\
\text { Out- } \\
\text { patients }\end{array}$ & 52 & $34.7(10.7)$ & $12.3(3.2)$ & $0.3(0.7)$ & $4.7(5.3)$ \\
& 52 & $37.4(10.4)$ & $10.8(4.0)$ & $18.3(5.6)$ & $33.5(13.2)$ \\
\end{tabular}

differences in nervous system response, and emotional response. In order to obtain useful data, we need to choose appropriate emotional induction methods. Using the classic oddball experimental paradigm of psychology (Li, 2014), we designed 5 stimuli tasks of 3 emotional valences to induce behavioral differences between healthy and depressed individuals. The tasks included:

1. Watching film clips: Three short film segments around $90 \mathrm{~s}$ each, one positive, another neutral, and the other negative, were disciplinarily presented. Participants were asked to watch the film clip and then describe their mood. The clips had previously been rated for their affective content (Gross and Levenson, 1995). The positive film clip is excerpted from cartoon "Larva Funny Bugs," the neutral film clip is excerpted from the documentary "Universe Millennium," and the negative film clip is excerpted from the movie "October Siege." The synchronized start of the stimuli with the recording enabled us to draw a correlation between facial activity and the stimuli.

2. Replying to nine free-response questions: Each participant was requested to respond to nine specific questions (three positives, three neutrals, and three negatives). These questions are designed based on DSM-IV and other depression scales such as the Hamilton depression rating scale (HDRS) ${ }^{2}$. Questions included, for instance, "what kind of lifestyle do you like?" "discuss a sad childhood memory," and "please evaluate yourself." The answers were synchronized with the facial activity recorded.

3. Reading three phonetically balanced passages containing affective content: The participants were presented with a paragraph of text on a computer screen and asked to finish the reading as naturally as possible. There are three reading materials. One of passages contained positive words (e.g., glorious, victory), and the other contained negative words (e.g., heart-broken, pain), which were selected from affective the ontology corpus created by Hongfei $\operatorname{Lin}^{3}$. The last one included neutral words (e.g., village, center) selected from the extremum table of affective Chinese words (Gong et al., 2011). The reading and recording commenced synchronously.

4. Describing pictures: The picture description section is to present 6 pictures in sequence on the computer screen. The first three pictures are facial expression pictures of three women divided into positive, neutral, and negative, and the last three pictures are three scene pictures divided into positive, neutral, and negative. All pictures were selected from the Chinese Facial Affective Picture System (CFAPS) (Gong et al., 2011). Participants were requested to observe the picture and then describe it. Reporting logs enabled correlations between image presentations and facial activity to be established.

\subsubsection{Process of Affective Rating}

The Self-Assessment Manikin (SAM) (Lang, 1980) which is an affective rating-scale system using a graphical figure that depicts

\footnotetext{
${ }^{2}$ Available at: http://ir.dlut.edu.cn/Group.aspx? $\mathrm{ID}=4$

${ }^{3}$ Available at: http://www.datatang.com/data/43216.
} 


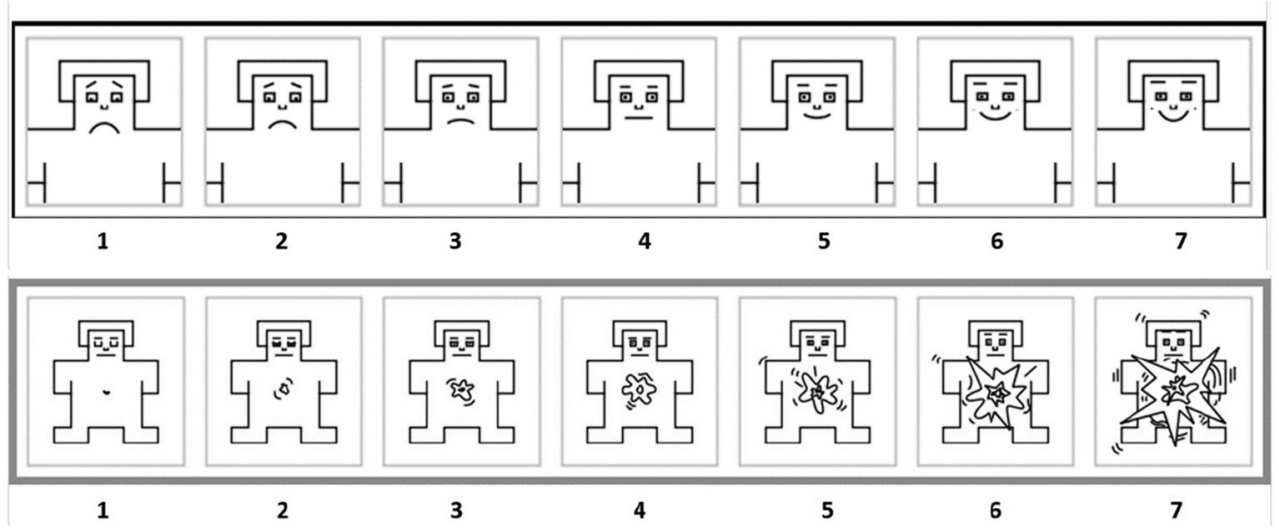

FIGURE 6 | Schematic diagram of affective rating. The top is valence rating. The bottom is arousal rating.

the dimensions of valence (from a smiling figure to a frowning figure) and arousal (from an excited to a relaxed figure), is used to measure a participant's emotion, as shown in Figure 6. The affective rating process consists of three parts: emotional pretest, emotion-eliciting tasks, and emotional posttest.

\subsubsection{Constructing Depression Dataset}

Every participant has to complete five stimulus tasks of three emotional valences in turn, resulting in 15 datasets: three datasets for watching film clips (one positive + one neutral + one negative), three datasets for nine interviews (three positive + three neutral + three negative), three datasets for text readings (one positive + one neutral + one negative), three datasets for expression image descriptions (one positive + one neutral + one negative), and three datasets for scene image descriptions (one positive + one neutral + one negative). The database consists of four folders for each participant, which are voice, video, emotional state, and information log. Fifteen monophonic speech recordings are made in the voice folder. A sampling rate of 44.1 $\mathrm{kHz}$ and a sampling depth of 24-bit are used for collecting speech signals. Speech recordings are saved in the uncompressed WAV format. Ambient noise should be lower than $60 \mathrm{~dB}$. There are two types of data in the video folder. One is 15 video recordings of $640 \times 480$ pixel, $30 \mathrm{fps}$ collected by a webcam, and saved as mp4; the other is 15 recordings obtained by the Kinect, and each recording contains two kinds of data: three-dimensional coordinates of 1,347 facial points and 17 AUs. Every facial point is a $3 \mathrm{D}$ point with $\mathrm{X}, \mathrm{Y}$, and $\mathrm{Z}$ coordinates. Figure 7 shows that facial contours consist of 1,347 feature points. At present, many AU detection methods use feature point tracking, shape modeling, template matching, and neural network to recognize the $\mathrm{AU}$ features of the face. In this paper, the Kinect device automatically recognizes the $\mathrm{AU}$ of the face through the builtin API interface and the facial AU detection algorithm. The intensity of each AU in each frame was calculated. The intensity amplitude was between -1 and 1 , and the facial expression was directly measured by digital features. Seventeen AUs recorded by Kinect are corresponding to AUs encoded by FACS. AU from

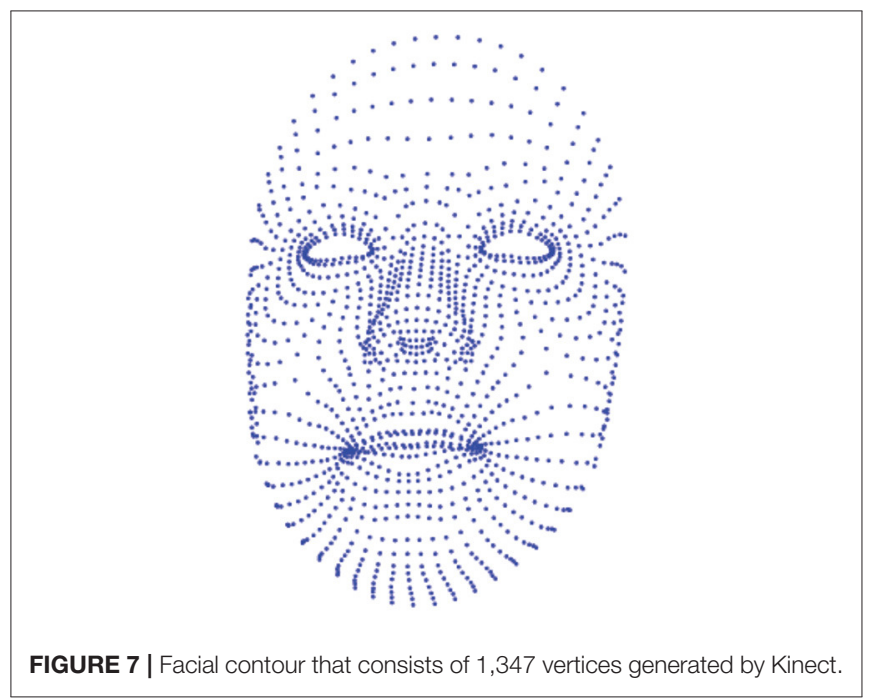

Kinect can appear separately or in combination to show different expressions ${ }^{4}$. Fifteen three-dimensional facial points recordings and 15 AUs recordings are saved as CSV format. Participants are assessed for the two dimensions of valence and arousal before and after receiving different emotion-eliciting tasks to obtain 15 evaluation results, which are saved in the emotional state folder. The information related to the subjects is saved in the information log, which are name, gender, age, profession, education background, label, PHQ-9, BDI, and so on.

\subsection{Data Pre-processing}

We intercepted the data which the subjects did not speak during the three tasks of watching the film clips, facial expression pictures description, and scene pictures description. The machine learning toolkit Dlib (King, 2009) is used to acquire facial region cropped and aligned according to each video's eye location. All images are resized to $100 \times 100$. Considering the problem of

\footnotetext{
${ }^{4}$ Available at: https://blog.csdn.net/u014365862/article/details/48212757.
} 
many frame redundancy in the video clip, we adopt the sampling scheme of taking one frame every 100 frames. Experiments determine the sampling interval according to the number of frames or length of each video and the frequency of facial changes. Finally, we obtained 156,000 facial image data in any given stimulus task. Then, we adopt the same sampling strategy on the Kinect dataset to ensure that the two data are aligned on the time axis. These data were used for training, validation, and testing networks. The original image is then divided into three images by $\mathrm{R}, \mathrm{G}$, and $\mathrm{B}$ channels and used to train a DBN. Every image is flatted, and then the pixel value of which is normalized for input in $2 \mathrm{D}-\mathrm{SADN}$. In 3DDGDN, three-dimensional coordinates of 1,347 facial points can be considered as one-dimension vector at frame $t$ and defined as $\left[x_{1}^{(t)} y_{1}^{(t)} z_{1}^{(t)} \cdots x_{n}^{(t)} y_{n}^{(t)} z_{n}^{(t)}\right]^{T}$, where $n$ is the total number of landmark points at frame $t$. I - score standardization is used to normalize xyz-coordinates as follows:

$$
\bar{x}_{i}^{(t)}=\frac{x_{i}^{(t)}-\mu}{\sigma}
$$

where $x_{i}^{(t)}$ is $\mathrm{x}$-coordinate of the $i$ th facial landmark point at frame $t, \mu$, and $\sigma$ is mean value and standard deviation of $\mathrm{x}$-coordinate at frame $t$, respectively. This process is also applied to $y_{i}^{(t)}, z_{i}^{(t)}$, and AUs. Finally, these normalized points are concatenated $\left[\bar{x}_{1}^{(t)} \bar{y}_{1}^{(t)} \bar{z}_{1}^{(t)} \cdots \bar{x}_{n}^{(t)} \bar{y}_{n}^{(t)} \bar{z}_{n}^{(t)}\right]^{T} \quad$ or $\left[\bar{x}_{1}^{(t)} \bar{y}_{1}^{(t)} \bar{z}_{1}^{(t)} \cdots \bar{x}_{n}^{(t)} \bar{y}_{n}^{(t)} \bar{z}_{n}^{(t)} A U_{1}^{(t)} \cdots A U_{17}^{(t)}\right]^{T}$ to input the DBN.

\subsection{Network Architecture}

The DBNs structure of 2D-SADN and 3D-DGDN are similar. The first layer RBM is trained completely unsupervised for all DBNs. The biases and weights are randomly sampled from a normal distribution with $\mu=0, \sigma=0.01$. They are all updated after a full minibatch. Because the preprocessed data is larger in $2 \mathrm{D}-\mathrm{SADN}$, a penalty term is added to the weight and bias updates to obtain sparse representation. $\lambda$ is fixed as 3 , the sparsity parameter of bias is 0.1 , and the learning rate is 0.02 . The rest RBMs are also trained for 100 epochs using the same value used for training the first level RBM for all DBNs.

The hidden nodes number of every channel DBN is fixed to 8192-4096-2048-502 in 2D-SADN. The number of hidden nodes for $4 \mathrm{DBN}$ and $4 \mathrm{DBN}-\mathrm{AU}$ from the first layer to 4-layer is selected over 3000-2048-1024-128 in 3D-DGDN. However, for AU-4DBN, the penultimate hidden layer with 1,024 nodes similar to $4 \mathrm{DBN}$ is then concatenated with $\mathrm{AUs}$, and the resulting input serves as the visible layer of a top-level RBM with 150 hidden nodes (Gaussian-Bernoulli).

Our LSTM has two layers, one with 200 nodes and another with 64 nodes. We initialize the hidden states to zero and then use the current minibatch's final hidden states as the initial hidden state of the subsequent minibatch. The batch size is 50 , and the training epoch is 50 . The learning rate is set by grid search, and the momentum is 0.9 .

The whole system is tested on the TensorFlow deep learning framework with a Xeon(R) CPU E7-4820 v4@2.00 GHz
TABLE 2 | The differences in valence and arouse dimension between the healthy and depressed group for the five stimuli with three emotional valences $(P<0.1)$.

\begin{tabular}{|c|c|c|c|c|c|}
\hline $\begin{array}{l}\text { Emotional } \\
\text { dimension }\end{array}$ & Tasks & Subjects & Positive & Neutral & Negative \\
\hline & & Health & 1.635 & 0.074 & -1.534 \\
\hline & Film & depression & -1.058 & -0.036 & -1.078 \\
\hline & & $P$-value & 0.043 & 2.428 & 0.052 \\
\hline & & Health & 1.356 & 0.088 & -0.744 \\
\hline & Question & Depression & -0.273 & 0.333 & -0.330 \\
\hline & & $P$-value & 0.601 & 0.565 & 1.035 \\
\hline & & Health & 0.947 & 0.260 & 0.829 \\
\hline \multirow[t]{15}{*}{ Valence } & Reading & Depression & 0.273 & 0.242 & 0.333 \\
\hline & & $P$-value & 0.233 & 0.137 & 0.531 \\
\hline & & Health & 1.084 & 0.205 & -0.938 \\
\hline & Expression figure & Depression & -0.152 & -0.198 & -0.506 \\
\hline & & $P$-value & 0.073 & 0.960 & 0.085 \\
\hline & & Health & 0.874 & 0.110 & -0.123 \\
\hline & Scene figure & Depression & -0.015 & 0.061 & 0.303 \\
\hline & & $P$-value & 0.125 & 1.531 & 0.211 \\
\hline & & Health & 1.058 & -0.205 & 1.045 \\
\hline & Film & Depression & -0.635 & 0.076 & 0.014 \\
\hline & & $P$-value & 0.072 & 0.151 & 0.065 \\
\hline & & Health & 0.968 & 0.027 & 0.109 \\
\hline & Question & Depression & -0.060 & -0.030 & 0.151 \\
\hline & & $P$-value & 0.254 & 0.096 & 0.325 \\
\hline & & Health & -0.810 & 0.137 & 0.164 \\
\hline \multirow[t]{8}{*}{ arousal } & Reading & Depression & -0.182 & -0.106 & 0.076 \\
\hline & & $P$-value & 0.222 & 0.115 & 0.177 \\
\hline & & Health & 1.008 & 0.205 & 0.233 \\
\hline & Expression figure & Depression & -0.014 & 0.333 & 0.106 \\
\hline & & $P$-value & 0.098 & 0.042 & 0.087 \\
\hline & & Health & 0.219 & 0.055 & -0.068 \\
\hline & Scene figure & Depression & 0.015 & -0.091 & -0.121 \\
\hline & & $P$-value & 0.146 & 0.033 & 0.088 \\
\hline
\end{tabular}

The table's data are the statistical value of the difference between the pretest and posttest of the valence dimension and arouse dimension under different stimuli tasks. Bold indicates significant difference.

processor, 128 Gigabytes memory, and a Telsa M60 GPU, which can meet our computing needs.

\subsection{Experimental Results}

\subsubsection{Qualitative Analysis of Stimulus Tasks}

Previous studies have shown that depressed patients have less positive emotions and more negative emotions than healthy individuals, which indicates that depressed patients show sad when stimulated by positive or negative emotions (DelleVigne et al., 2014). Depressed patients will be less sensitive to emotional stimuli from the outside world; that is to say, it is difficult for depressed patients to elicit corresponding emotional feedback (Rottenberg, 2005). The differences in valence and arousal dimensions between the healthy and depressed groups for the five stimuli with three emotional valences were calculated, respectively, as shown in Table 2 . 
From Table 2, we can find that the absolute value of the healthy group's valence difference is generally greater than that of the depressed group in all stimulus tasks. The valence difference of the healthy group is basically consistent with emotional valence, which means that positive tasks stimulate joyful emotions, negative tasks stimulate sad emotions for the healthy group (the valence difference in positive stimuli is positive, and the valence difference in negative stimuli is negative), but for the depressed group, both positive and negative stimuli basically arouse sad emotions (the valence difference in positive and negative stimuli is negative). From the $T$-test values, it can be found that there is a significant difference in valence between the healthy group and depressed group under positive and negative stimulation, especially in the stimulation tasks of film clips and characters' facial expressions, as shown in bold.

From Table 2, we also can find that the arousal difference of the healthy group is almost greater than that of the depressed group in all stimulus tasks, and the arousal difference of the healthy group is basically positive, which means that the healthy group is more likely to be aroused than the depressed group. From the $T$-test values, it can be found that there is a significant difference in arousal between the healthy group and depressed group under positive and negative stimulation, especially in the stimulation tasks of film clips and characters' facial expressions, as shown in bold.

From Table 2, we can draw the following conclusions: positive and negative film clips and facial expression pictures are more likely to inspire significant differences between the healthy and depressed groups than the other three tasks. Moreover, the results reflected from Table 2 are also consistent with paper (DelleVigne et al., 2014) and (Rottenberg, 2005). In order to more intuitively reflect the effectiveness of the experimental paradigm we designed, we draw comparison charts of the valence and arousal of the healthy group and depressed group before and after positive film clips stimulation, as shown in Figure 8. The healthy group was in a calm mood before watching the positive film clip, and the pleasure degree increased significantly after watching the film clip, which stimulated a happy mood. The depressed group felt a little sad before watching the film clip, but their mood became more and more depressed after watching the film clip, and the arousal degree did not change much. This is consistent with the characteristics of depression.

\subsubsection{Determining the Number of Network Layers}

We use three kinds of data in the whole framework, namely 2D images, 3D facial landmark points, and AUs. We first use $2 \mathrm{D}$ face images and 3D facial landmark points as input to train different deep DBNs for the five stimuli with three emotional valences, respectively. We use the validation set to test networks and find that the recognition accuracy of the both mainly increases with the number of layers, reaching the highest on the forth hidden layer using 2D images or 3D landmark points trained DBN models, but both of them subsequently lose recognition performance as the number of layers increases, as shown in Figure 9. Therefore, we regard 4-hidden-layer DBN as a benchmark model of the 3D-DGDN.

\subsubsection{Global Performance Analysis}

The accuracy of all proposed models for three emotional valences of the five stimuli was tested on the dataset, including all males and females. The experiment results are shown in Table 3 and Figure 10. It can be seen from Table 3 and Figure 10 that the best performance among the three emotional valences of the five stimuli was obtained based on the joint model. In particular, the performance of 3D-DGDN was higher than the $2 \mathrm{D}-\mathrm{SADN}$ in all tasks. In the process of data collection, it was found that depressed patients or subjects with depressive tendencies were more prone to hyperactivity, which would lead to changes in depth information. Therefore, we added time series information to depth information for modeling, which will obtain more discriminative features. However, the combined network produced good results. This indicates that the 2D-SADN

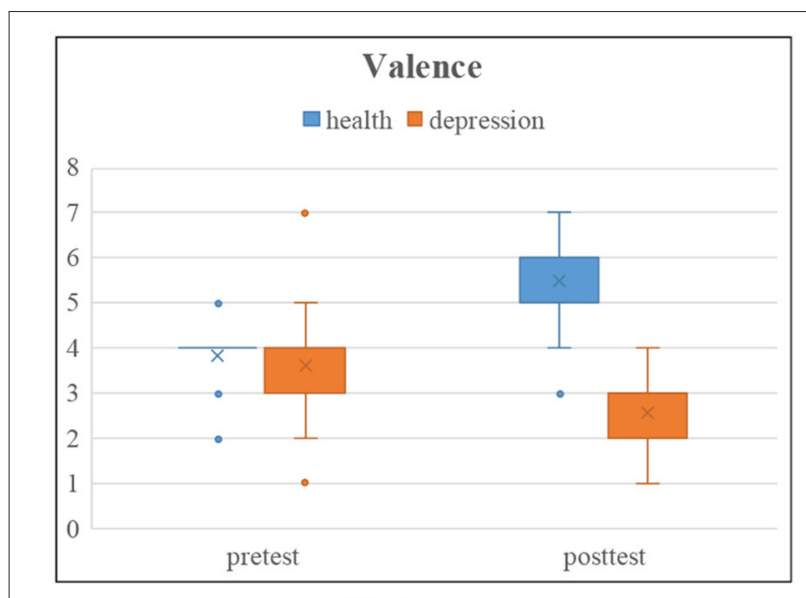

Valence

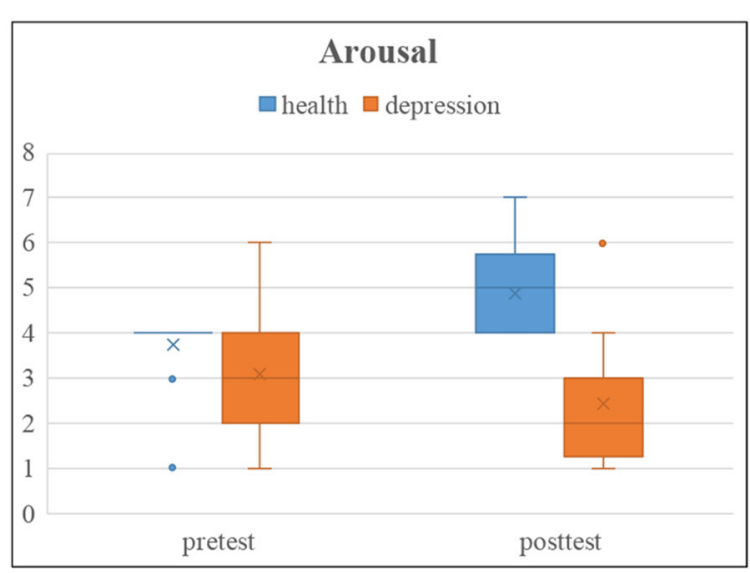

Arousal

FIGURE 8 | Box comparison charts of the valence and arousal of the healthy group and depressed group before and after positive film clips stimulation. 


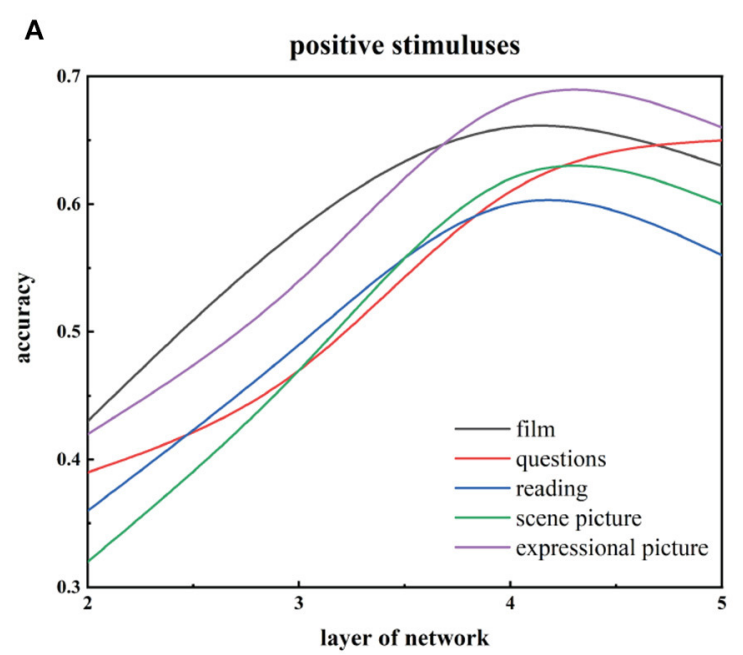

B

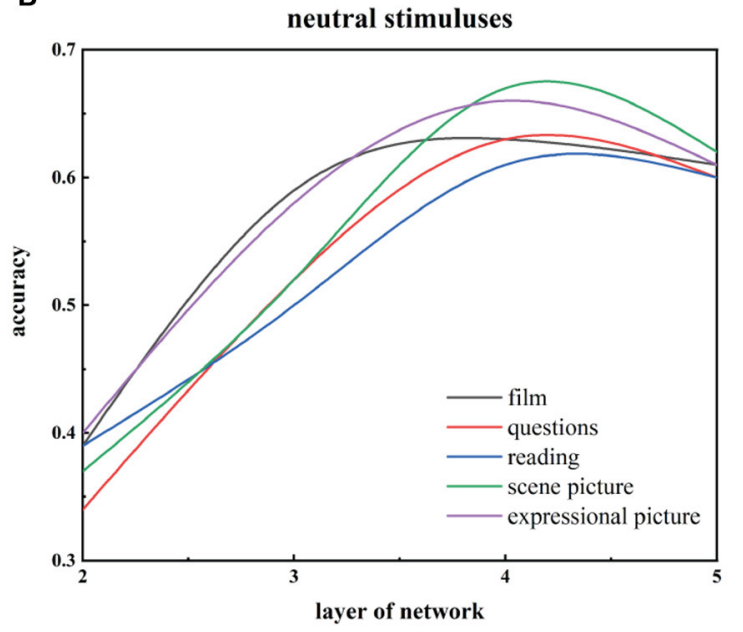

C

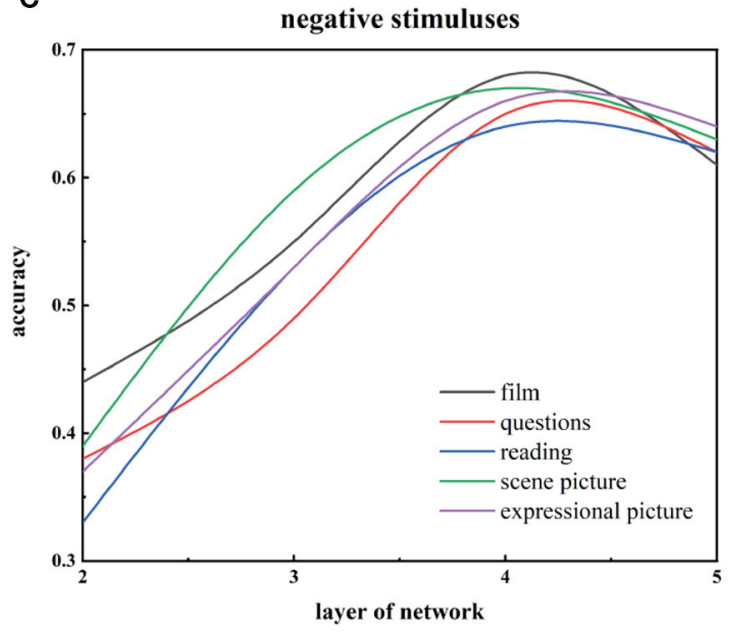

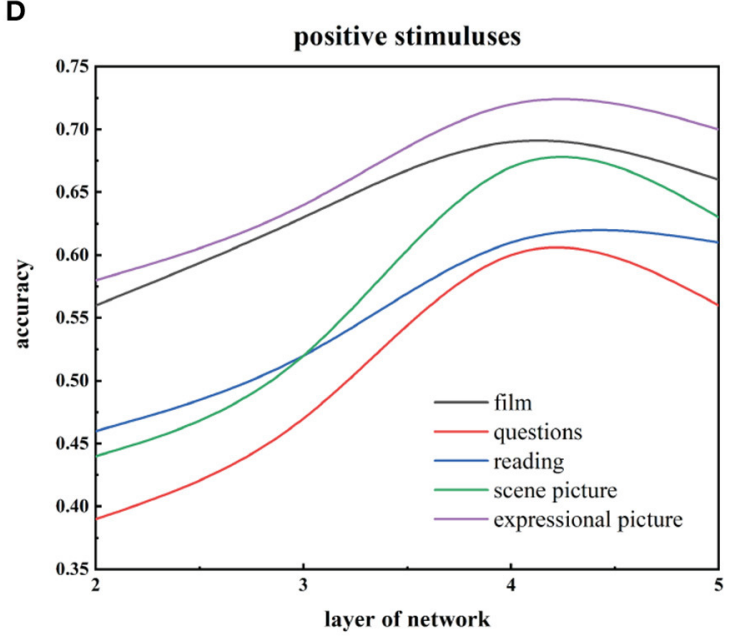

E
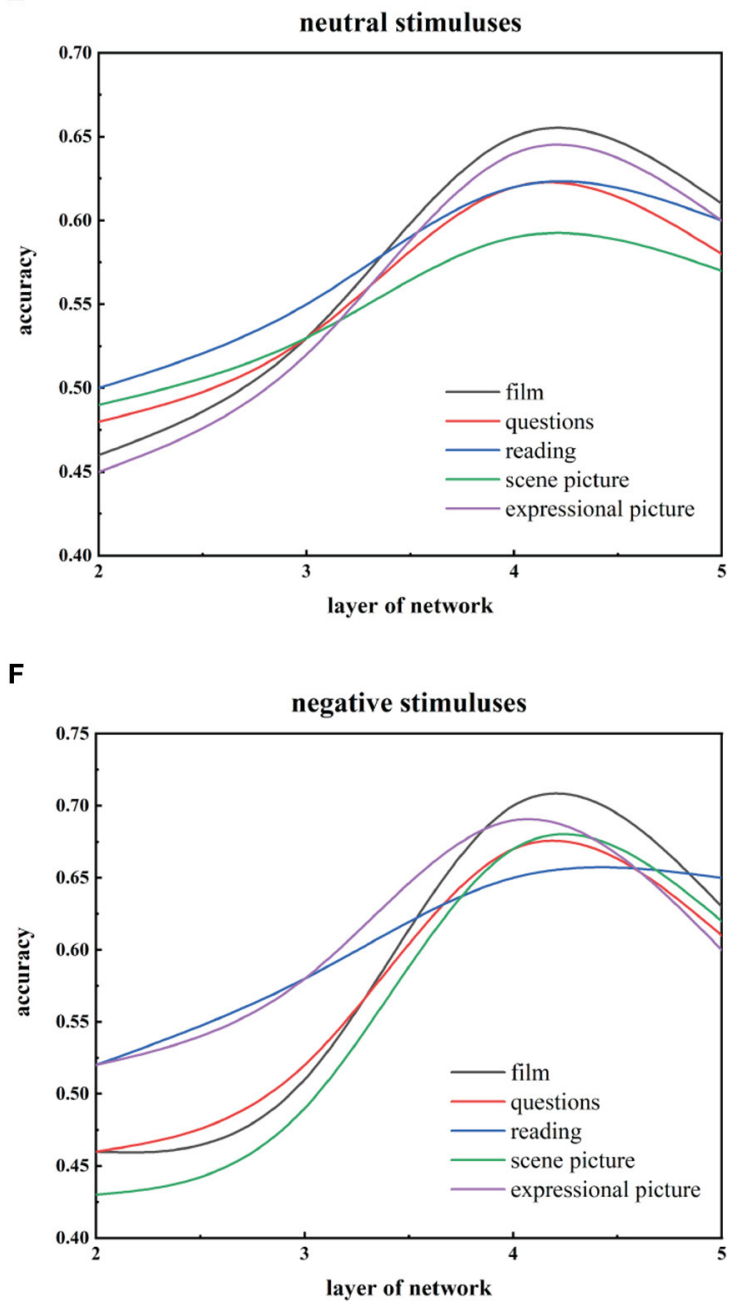

FIGURE 9 | Comparison of recognition performance with the different hidden levels on three emotional valences of the five stimuli for 3D facial points and 2D images. (A-C) are based on 3D facial points. (D-F) are based on 2D images. 
TABLE 3 | The accuracy of all models for three emotional valences of five stimuli.

\begin{tabular}{|c|c|c|c|c|c|}
\hline Type & Models & Positive & Neutral & Negative & Mean \\
\hline & 4DBN-AU & 0.638 & 0.603 & 0.673 & 0.638 \\
\hline & $\mathrm{AU}-4 \mathrm{DBN}$ & 0.693 & 0.635 & 0.701 & 0.676 \\
\hline \multirow[t]{5}{*}{ Film } & 3D-DGDN & 0.745 & 0.677 & 0.752 & 0.725 \\
\hline & 2D-SADN & 0.682 & 0.617 & 0.694 & 0.664 \\
\hline & Joint(2D-3D) & 0.798 & 0.716 & 0.807 & 0.774 \\
\hline & 4DBN-AU & 0.605 & 0.593 & 0.592 & 0.597 \\
\hline & AU-4DBN & 0.639 & 0.636 & 0.642 & 0.639 \\
\hline \multirow[t]{5}{*}{ Question } & 3D-DGDN & 0.687 & 0.659 & 0.693 & 0.680 \\
\hline & 2D-SADN & 0.618 & 0.632 & 0.647 & 0.632 \\
\hline & Joint(2D-3D) & 0.702 & 0.683 & 0.713 & 0.699 \\
\hline & 4DBN-AU & 0.572 & 0.583 & 0.601 & 0.585 \\
\hline & $\mathrm{AU}-4 \mathrm{DBN}$ & 0.623 & 0.625 & 0.652 & 0.633 \\
\hline \multirow[t]{5}{*}{ Reading } & 3D-DGDN & 0.668 & 0.658 & 0.694 & 0.673 \\
\hline & 2D-SADN & 0.583 & 0.613 & 0.635 & 0.610 \\
\hline & Joint(2D-3D) & 0.711 & 0.697 & 0.712 & 0.707 \\
\hline & 4DBN-AU & 0.617 & 0.538 & 0.608 & 0.588 \\
\hline & $\mathrm{AU}-4 \mathrm{DBN}$ & 0.671 & 0.592 & 0.672 & 0.645 \\
\hline \multirow[t]{5}{*}{ Scene picture } & 3D-DGDN & 0.716 & 0.651 & 0.724 & 0.697 \\
\hline & 2D-SADN & 0.623 & 0.613 & 0.668 & 0.635 \\
\hline & Joint(2D-3D) & 0.747 & 0.707 & 0.752 & 0.735 \\
\hline & 4DBN-AU & 0.659 & 0.591 & 0.635 & 0.628 \\
\hline & $\mathrm{AU}-4 \mathrm{DBN}$ & 0.703 & 0.642 & 0.690 & 0.678 \\
\hline \multirow[t]{3}{*}{ Expression picture } & 3D-DGDN & 0.729 & 0.683 & 0.751 & 0.721 \\
\hline & 2D-SADN & 0.684 & 0.657 & 0.668 & 0.700 \\
\hline & Joint(2D-3D) & 0.770 & 0.725 & 0.783 & 0.759 \\
\hline
\end{tabular}

Bold indicates a higher recognition rate.

network is a performance supplement to the 3D-DGDN network, and the two networks are complementary to each other.

It can be also seen that the recognition accuracy of positive and negative stimuli is higher than that of neutral stimulus, which is consistent with the emotional feedback theory of depressed patients compared with the healthy group, patients with depression have behavioral patterns such as weakened positive emotional feedback and enhanced negative emotional feedback. So in some cases, they have formed specific facial expressions such as reduced positive expressions and increased negative expressions (Babette et al., 2005). That is to say, they will not produce a larger change in expression compared with the normal population when facing the same stimulus. Therefore, there is a significant difference between positive and negative stimuli. Simultaneously, the accuracy of watching film clips is relatively higher in all positive or negative stimulus tasks and the highest recognition rates reach up to 0.798 and 0.807 for positive and negative stimulus, respectively, as shown in bold. The next high recognition rate is to view the expressional picture, as shown in bold. Because emotionally charged clips and images can, in principle, elicit an observable response (Pampouchidou et al., 2019). It is because that in order to eliminate the influence of unrelated facial movements on the facial expression behavior analysis of the participants, we only used the experimental data that the participants are completely prohibited from speaking in these two tasks. Relatively poor accuracies are obtained for questions and readings, which could be because facial expressions are associated with speech. When one feature is mixed with other factors, the purity expressed by this feature is not high.

\subsubsection{Difference in Gender Analysis}

The gender-dependent experiments are analyzed based on the integrated network. Figure 11 shows the comparison of depression recognition based on gender difference under $95 \%$ confidence interval. From Figure 11, we can find that females' recognition accuracies are universally higher than that of males in three kinds of emotion valence, which explains that females are more likely to be aroused emotionally. According to $\mathrm{WHO}$, evidence suggests that women are more prone than men to experience anxiety, depression, and somatic complaintsphysical symptoms that cannot be explained medically ${ }^{5}$. We can also see that women are more likely to show the effects of positive stimulation than men. Among the three emotional valence tasks, the difference between female and male groups under the negative stimuluses are the smallest, which indicates that both female and male groups have higher accuracy and sensitivity under the negative stimuluses. In general, female are more emotionally aroused than male.

\subsubsection{Comparative Analysis of Relevant Works}

We compared our method's accuracy with the methods using the same data set, as shown in Table 4. Although our accuracy rate was slightly lower than that in previous studies ( $\mathrm{Li}$ et al., 2018) (the selected samples are all major depressive disorder) in neutral stimulation tasks, we have improved the accuracy in the remaining tasks. Studies have shown that severely depressed individuals have lower emotional feedback to both positive and negative stimuli compared with healthy individuals (Nesse and Randolph, 2000). Therefore, our research results are more in line with the emotional experience of depressed patients. However, the performance has declined compared with our previous work (Guo et al., 2019). We will compare and summarize from the following points:

- Data source: The previous work only used the threedimensional face point data collected by Kinect, although the point data can better handle some additional variables in the picture, such as illumination, angle, skin color, and so on, the videos (pictures) collected by the optical camera are still the main focus in actual application scenarios. Therefore, we combined the two data sources to make up for each other in this work.

- Model: The main framework of the DBN model was used in the two works. Considering that it was an extension of the previous work, the same data samples and the main model framework were used. However, this work further upgraded the model, using DBN to extract static facia appearance information and combined with the LSTM network to extract the dynamic information, and finally through a full connection

\footnotetext{
${ }^{5}$ Available at: https://www.who.int/life-course/news/commentaries/2015-intlwomens-day/.
} 


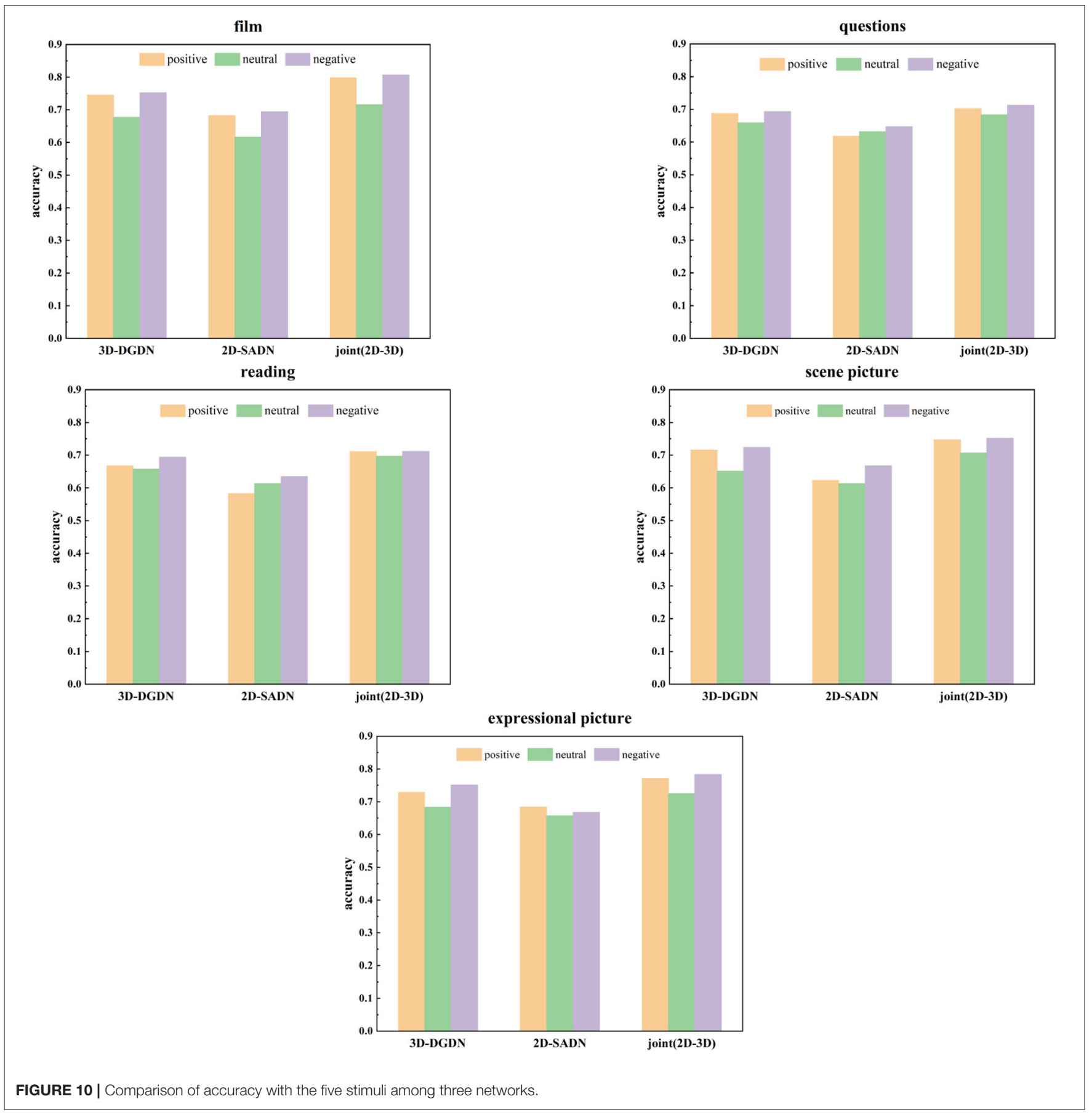

to connect the two networks. The design of the model is complete and more prosperous than the previous work.

- Practice: We sampled frames by frames in the previous work, preprocessed 3D facial points data, and converted them into $200 * 200$ grayscale images. It took nearly a week to calculate the entire batch of data for 30 cycles. In this work, we sampled between frames and processed the original data directly. Although the network was more complicated than previous work, the calculation time was reduced. It only took 5 days to complete the whole batch of data for 100 cycles.
However, the classification accuracy has declined due to the following aspects.

First of all, the previous work results showed that visual stimuli classification effect, such as watching film clips and pictures, was better than the classification effect of language expression (here, mainly talking about changes in facial expressions). In our entire experiment, subjects completed every task and were asked to answer questions. The data used earlier included responses to questions on watching the film clips and seeing facial/scene expression tasks. In this work, to further verify 


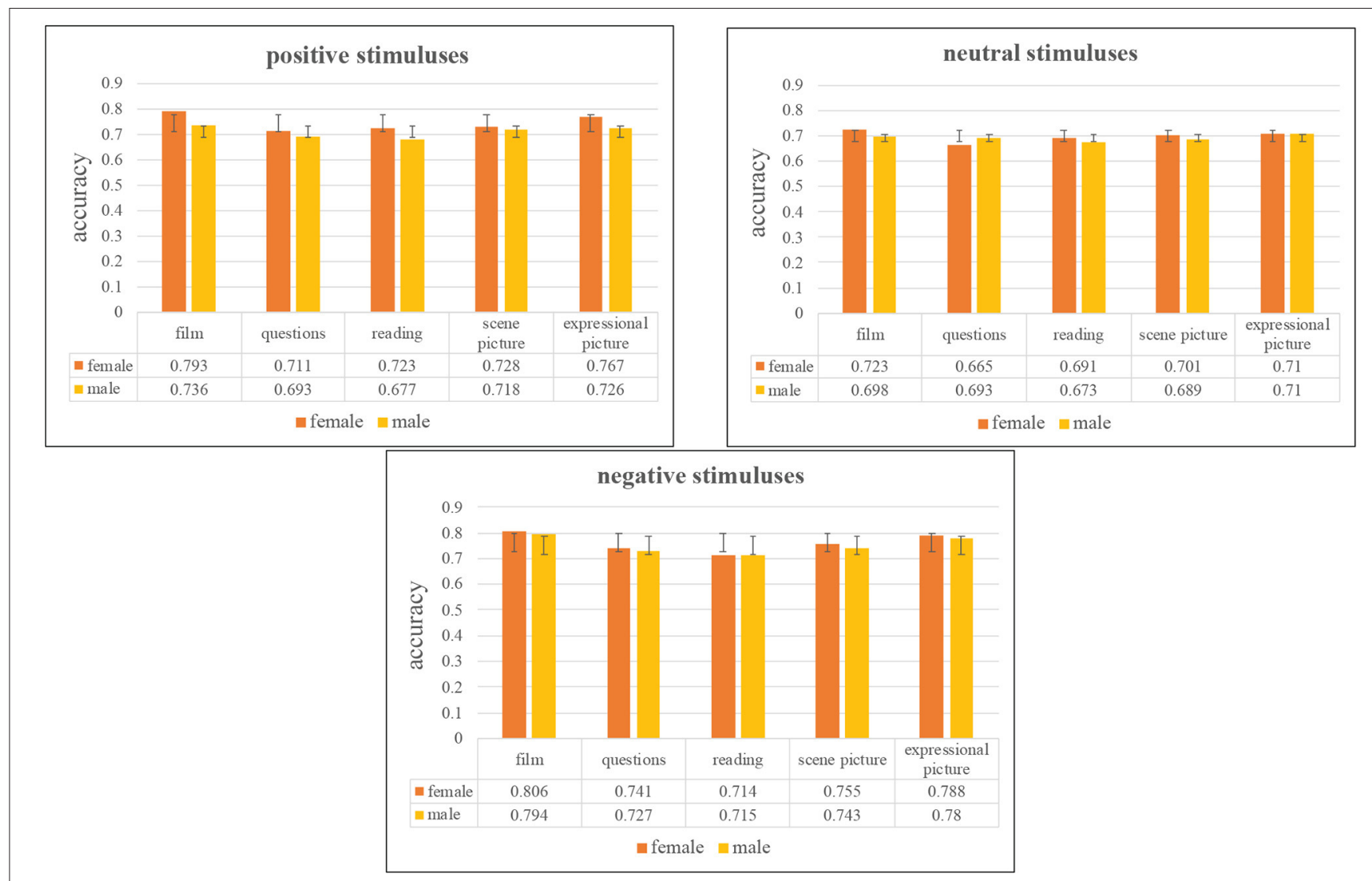

FIGURE 11 | Comparison of accuracy on different gender under 95\% confidence interval.

whether direct visual stimuli are more likely to elicit emotions in the depressed group, we selected only the data participants did not speak during watching the film clips and seeing facial/scene expression tasks. Coupled with the strategy of sampling between frames, the overall amount of data is far less than the previous work. For deep learning, the more considerable the amount of data, the better the trained model's performance.

Then, we converted 1,347 three-dimensional points into $200 * 200$ grayscale images in the preliminary work, which undoubtedly added some additional information for recognition. Whether this information played a role in the performance of the model could not be reasonably explained. In this work, we directly used the raw data without any additional information, so the final results are calculated from both the data and the model.

Besides, this paper's conclusion was obtained after 100 cycles based on 10-fold cross-validation, which is more stable than previous work.

In conclusion, on the basis of using the original data, the study in this paper significantly reduces the calculation time, while the accuracy rate is slightly lower than before, such as 0.02 for women and 0.01 for men. Based on the above points, this work is more reasonable and universal from three aspects of theory, model, and practice.

\section{CONCLUSION AND FUTURE WORK}

Depression is a common mental illness that can negatively affect people's mental health and daily life. In recent years, researchers have been looking for an objective evaluation method and quantitative indicators to identify depression objectively and effectively. Among them, the research of depression recognition based on facial expression behavior is a hot topic. In this paper, We first designed an experimental paradigm that can effectively stimulate the emotional differences between healthy and depressed groups and established a database for identifying depression. And then, we presented two deep network models that collaborate with each other. The first network was 2DSADN, which is used to extract the static appearance features from images, and the second network was 3D-DGDN, which captures the dynamic geometry features of 3D facial landmark points and AUs from Kinect. We showed that the accuracy obtained by the 2D-SADN was lower than that of the 3D-DGDN, which may be because poor image quality and the DBN model cannot well retain the $2 \mathrm{D}$ information of an image. At last, we achieved the best recognition rates using the integrated deep network on the collected databases.

From the perspective of emotional stimulus materials, the experimental results also support this theory: apparent 
TABLE 4 | Comparison of accuracy based on the same database.

\begin{tabular}{|c|c|c|c|c|c|c|c|c|c|c|}
\hline \multirow[t]{3}{*}{ Gender } & \multirow[t]{3}{*}{ Task } & \multicolumn{9}{|c|}{ Accuracy } \\
\hline & & \multicolumn{3}{|c|}{ Positive stimulus } & \multicolumn{3}{|c|}{ Neutral stimulus } & \multicolumn{3}{|c|}{ Negative stimulus } \\
\hline & & $\begin{array}{l}\text { This } \\
\text { work }\end{array}$ & $\begin{array}{l}\text { Previous } \\
\text { work }\end{array}$ & (Li et al., 2018) & $\begin{array}{l}\text { This } \\
\text { work }\end{array}$ & $\begin{array}{l}\text { Previous } \\
\text { work }\end{array}$ & (Li et al., 2018) & $\begin{array}{l}\text { This } \\
\text { work }\end{array}$ & $\begin{array}{l}\text { Previous } \\
\text { work }\end{array}$ & (Li et al., 2018) \\
\hline \multirow{5}{*}{ Female } & Film clips & 0.793 & 0.825 & 0.737 & 0.723 & 0.761 & 0.868 & 0.806 & 0.816 & 0.763 \\
\hline & Questions & 0.711 & 0.761 & 0.649 & 0.665 & 0.705 & 0.737 & 0.741 & 0.765 & 0.675 \\
\hline & Readings & 0.723 & 0.768 & 0.711 & 0.691 & 0.728 & 0.658 & 0.714 & 0.751 & 0.658 \\
\hline & Scene pictures & 0.728 & 0.801 & 0.711 & 0.701 & 0.713 & 0.763 & 0.755 & 0.806 & 0.711 \\
\hline & $\begin{array}{l}\text { Expression } \\
\text { pictures }\end{array}$ & 0.767 & 0.806 & 0.632 & 0.710 & 0.741 & 0.737 & 0.788 & 0.801 & 0.711 \\
\hline \multirow{5}{*}{ Male } & Film clips & 0.736 & 0.772 & 0.647 & 0.698 & 0.733 & 0.794 & 0.794 & 0.782 & 0.647 \\
\hline & Questions & 0.693 & 0.738 & 0.725 & 0.693 & 0.728 & 0.735 & 0.727 & 0.726 & 0.667 \\
\hline & Readings & 0.677 & 0.724 & 0.618 & 0.673 & 0.694 & 0.676 & 0.715 & 0.745 & 0.588 \\
\hline & Scene pictures & 0.718 & 0.755 & 0.618 & 0.689 & 0.714 & 0.706 & 0.743 & 0.776 & 0.647 \\
\hline & $\begin{array}{l}\text { Expression } \\
\text { pictures }\end{array}$ & 0.726 & 0.761 & 0.647 & 0.710 & 0.673 & 0.706 & 0.780 & 0.737 & 0.588 \\
\hline
\end{tabular}

differences existed between the health and depressed groups for pleasant or unpleasant stimuli. Mostly, the accuracy of watching film clips and expressional pictures emotional stimulus tasks were generally high, but the accuracy of answering questions and reading texts is low. This is because the subjects recorded facial expressions while speaking in both the masks, with one feature being mixed with other factors. We will further investigate the experimental strategies to construct a more distinctively characteristic depression behavior database in future work. We will further analyze which of the two states of speech and non-speech information on discriminating depressed patients. Since CNN has shown superior performance on image classification/recognition problem, we aim to use the CNN-based methods to model depth information and video information. We also will try to use the state-of-the-art multimodal fusion methods to identify depression.

\section{DATA AVAILABILITY STATEMENT}

The data analyzed in this study is subject to the following licenses/restrictions: Data involves privacy and has not been disclosed. Requests to access these datasets should be directed to Zhenyu Liu, liuzhenyu@lzu.edu.cn.

\section{ETHICS STATEMENT}

The studies involving human participants were reviewed and approved by Tianshui Third People's Hospital and Lanzhou University. The patients/participants provided their written informed consent to participate in this study.

\section{AUTHOR CONTRIBUTIONS}

All authors listed have made a substantial, direct, and intellectual contribution to the work. WG, $\mathrm{HY}$, and $\mathrm{BH}$ were responsible for the entire study, including study concepts and study design. WG, ZL, and YX contributed to the experimental paradigm design. WG and YX were responsible for collecting data. WG wrote the manuscript. HY helped WG draft the manuscript and modify the important content. All authors read and approved the final manuscript.

\section{FUNDING}

This work was supported in part by the National Key Research and Development Program of China (Grant No. 2019YFA0706200), in part by the National Natural Science Foundation of China (Grant Nos. 61802159, 61632014, 61627808, 61210010, 11664036, 62067008), in part by the National Basic Research Program of China (973 Program, Grant No. 2014CB744600), in part by the Program of Beijing Municipal Science \& Technology Commission (Grant No. Z171100000117005), in part by the High School Science and Technology Innovation Team Project of Gansu (Grant No. 2017C-03).

\section{ACKNOWLEDGMENTS}

The authors acknowledge all subjects and thank Director Wang and Director Ding from Tianshui Third People's Hospital for clinical diagnosis.

\section{SUPPLEMENTARY MATERIAL}

The Supplementary Material for this article can be found online at: https://www.frontiersin.org/articles/10.3389/fnins. 2021.609760/full\#supplementary-material 


\section{REFERENCES}

Alghowinem, S. (2015). Multimodal analysis of verbal and nonverbal behavior on the example of clinical depression (Ph.D. thesis). The Australian National University Canberra, ACT.

Alghowinem, S., Goecke, R., Wagner, M., Epps, J., Hyett, M., Parker, G., et al. (2018). Multimodal depression detection: fusion analysis of paralinguistic, head pose and eye gaze behaviors. IEEE Trans. Affect. Comput. 9, 478-490. doi: 10.1109/TAFFC.2016.26 34527

Alghowinem, S., Goecke, R., Wagner, M., Parker, G., and Breakspear, M. (2013). "Eye movement analysis for depression detection," in 2013 IEEE International Conference on Image Processing (Melbourne, VIC), 4220-4224.

Aly, S., Abbott, A. L., and Torki, M. (2016). "A multi-modal feature fusion framework for kinect-based facial expression recognition using dual kernel discriminant analysis (dkda)," in 2016 IEEE Winter Conference on Applications of Computer Vision (WACV) (Lake Placid, NY), 1-10.

Anis, K., Zakia, H., Mohamed, D., and Jeffrey, C. (2018). "Detecting depression severity by interpretable representations of motion dynamics," in 2018 13th IEEE International Conference on Automatic Face \& Gesture Recognition (FG 2018) (Xi'an: IEEE), 739-745.

Babette, E., Heyn, K., Gebhard, R., and Bachmann, S. (2005). Facial expression of emotions in borderline personality disorder and depression. J. Behav. Ther. Exp. Psychiatry 36, 183-196. doi: 10.1016/j.jbtep.2005.05.002

Bhatia, S. (2016). "Multimodal sensing of affect intensity," in Proceedings of the 18th ACM International Conference on Multimodal Interaction, ICMI '16 (New York, NY: Association for Computing Machinery), 567-571.

Bhatia, S., Hayat, M., Breakspear, M., Parker, G., and Goecke, R. (2017). “A videobased facial behaviour analysis approach to melancholia," in 2017 12th IEEE International Conference on Automatic Face Gesture Recognition (FG 2017) (Washington, DC), 754-761.

Carvalho, N., Laurent, E., Noiret, N., Chopard, G., Haffen, E., Bennabi, D., et al. (2015). Eye movement in unipolar depression and bipolar disorders: A systematic review of the literature. Front. Psychol. 6:1809. doi: $10.3389 /$ fpsyg.2015.01809

Cohn, J. F., Kruez, T. S., Matthews, I., Yang, Y., Nguyen, M. H., Padilla, M. T., et al. (2009). "Detecting depression from facial actions and vocal prosody," in 2009 3 rd International Conference on Affective Computing and Intelligent Interaction and Workshops (Amsterdam), 1-7.

Delle-Vigne, D., Wang, W., Kornreich, C., Verbanck, P., and Campanella, S. (2014). Emotional facial expression processing in depression: data from behavioral and event-related potential studies. Neurophysiol. Clin. 44, 169-187. doi: 10.1016/j.neucli.2014.03.003

Demakakos, P., Cooper, R., Hamer, M., Oliveira, C., Hardy, R., and Breeze, E. (2015). The bidirectional association between depressive symptoms and gait speed: evidence from the english longitudinal study of ageing (ELSA). PLoS ONE 8:e68632. doi: 10.1371/journal.pone.0068632

Fischer, A., and Igel, C. (2012). "An introduction to restricted boltzmann machines," in Progress in Pattern Recognition, Image Analysis, Computer Vision, and Applications (Buenos Aires: Springer), 14-36.

Girshick, R., Donahue, J., Darrell, T., and Malik, J. (2014). "Rich feature hierarchies for accurate object detection and semantic segmentation," in Proceedings of the IEEE Conference on Computer Vision and Pattern Recognition, 580-587.

Gong, B., Wang, Y., Liu, J., and Tang, X. (2009). "Automatic facial expression recognition on a single $3 \mathrm{~d}$ face by exploring shape deformation," in Proceedings of the 17th ACM International Conference on Multimedia, MM '09 (New York, NY: Association for Computing Machinery), 569-572. doi: 10.3969/j.issn.1000-6729.2011.01.011

Gong, X., Huang, Y., Wang, Y., and Luo, Y. (2011). Revision of the chinese facial affective picture system. Chinese Ment. Health J. 25, 40-46.

Greff, K., Srivastava, R. K., Koutník, J., Steunebrink, B. R., and Schmidhuber, J. (2016). Lstm: a search space odyssey. IEEE Trans. Neural Netw. Learn. Syst. 28, 2222-2232. doi: 10.1109/TNNLS.2016.2582924

Gross, J. J. and Levenson, R. W. (1995). Emotion elicitation using films. Cogn. Emot. 9, 87-108. doi: 10.1080/02699939508408966

Guo, W., Yang, H., and Liu, Z. (2019). "Deep neural networks for depression recognition based on facial expressions caused by stimulus tasks," in 2019 8th International Conference on Affective Computing and Intelligent Interaction Workshops and Demos (ACIIW) (Cambridge, MA: IEEE), 133-139.

Gupta, R., Malandrakis, N., Xiao, B., Guha, T., Van Segbroeck, M., Black, M., et al. (2014). "Multimodal prediction of affective dimensions and depression in human-computer interactions," in AVEC'14 (Orlando, FL), 33-40.

He, L., Jiang, D., and Sahli, H. (2015). "Multimodal depression recognition with dynamic visual and audio cues," in 2015 International Conference on Affective Computing and Intelligent Interaction (ACII) (Xian), 260-266.

He, L., Jiang, D., and Sahli, H. (2018). Automatic depression analysis using dynamic facial appearance descriptor and dirichlet process fisher encoding. IEEE Trans. Multimedia 21, 1476-1486. doi: 10.1109/TMM.2018.28 77129

Hinton, G. E., Osindero, S., and Teh, Y.-W. (2006). A fast learning algorithm for deep belief nets. Neural Comput. 18, 1527-1554. doi: $10.1162 /$ neco.2006.18.7.1527

Jan, A., Meng, H., Gaus, Y. F. B. A., and Zhang, F. (2018). Artificial intelligent system for automatic depression level analysis through visual and vocal expressions. IEEE Trans. Cogn. Dev. Syst. 10, 668-680. doi: 10.1109/TCDS.2017.2721552

Jiang, H., Hu, B., Liu, Z., Yan, L., Wang, T., Liu, F., et al. (2017). Investigation of different speech types and emotions for detecting depression using different classifiers. Speech Commun. 90, 39-46. doi: 10.1016/j.specom.2017. 04.001

Joshi, J., Dhall, A., Goecke, R., Breakspear, M., and Parker, G. (2012). "Neuralnet classification for spatio-temporal descriptor based depression analysis," in Proceedings of the 21st International Conference on Pattern Recognition (ICPR2012) (Tsukuba), 2634-2638.

Jung, H., Lee, S., Yim, J., Park, S., and Kim, J. (2015). “Joint fine-tuning in deep neural networks for facial expression recognition," in 2015 IEEE International Conference on Computer Vision (ICCV) (Santiago), 2983-2991.

King, D. E. (2009). Dlib-ml: a machine learning toolkit. J. Mach. Learn. Res. 10, 1755-1758. doi: 10.1145/1577069.1755843

Kroenke, K., and Spitzer, R. L. (2002). The phq-9: a new depression diagnostic and severity measure. Psychiatr. Ann. 32, 509-521. doi: 10.3928/0048-5713-20020901-06

Lang, P. (1980). "Behavioral treatment and bio-behavioral assessment: computer applications," in Technology in Mental Health Care Delivery Systems, 119-137.

Leyvand, T., Meekhof, C., Wei, Y., Sun, J., and Guo, B. (2011). Kinect identity: technology and experience. Computer 44, 94-96. doi: 10.1109/MC.2011.114

Li, J., Liu, Z., Ding, Z., and Wang, G. (2018). "A novel study for mdd detection through task-elicited facial cues," in 2018 IEEE International Conference on Bioinformatics and Biomedicine (BIBM) (Madrid: IEEE), 1003-1008.

Li, Y. (2014). An ERP study of cognitive processing of subthreshold depression individuals on different stimulus emotional pictures (Master's thesis). Beijing University of Chinese Medicine (Beijing).

Liu, M., Shan, S., Wang, R., and Chen, X. (2014). "Learning expressionlets on spatio-temporal manifold for dynamic facial expression recognition," in 2014 IEEE Conference on Computer Vision and Pattern Recognition (CVPR) (Columbus, $\mathrm{OH}$ ).

McIntyre, G., Göcke, R., Hyett, M., Green, M., and Breakspear, M. (2009). "An approach for automatically measuring facial activity in depressed subjects," in 2009 3rd International Conference on Affective Computing and Intelligent Interaction and Workshops (Amsterdam), 1-8.

McIntyre, G. J., et al. (2010). The computer analysis of facial expressions: on the example of depression and anxiety (Ph.D. thesis). The Australian National University. Canberra, ACT.

McPherson, A., and Martin, C. R. (2010). A narrative review of the beck depression inventory (bdi) and implications for its use in an alcohol-dependent population. J. Psychiatr. Ment. Health Nurs. 17, 19-30. doi: 10.1111/j.1365-2850.2009.01469.x

Melo, W. C. D., Granger, E., and Hadid, A. (2019). "Combining global and local convolutional 3d networks for detecting depression from facial expressions," in 2019 14th IEEE International Conference on Automatic Face \& Gesture Recognition (FG 2019) (Lille: IEEE), 1-8.

Meng, H., Huang, D., Wang, H., Yang, H., AI-Shuraifi, M., and Wang, Y. (2013). "Depression recognition based on dynamic facial and vocal expression features using partial least square regression," in Proceedings of the $3 r d A C M$ 
International Workshop on Audio/Visual Emotion Challenge, AVEC '13 (New York, NY: Association for Computing Machinery), 21-30.

Michalak, J., Troje, N. F., Fischer, J., Vollmar, P., Heidenreich, T., and Schulte, D. (2009). Embodiment of sadness and depression-gait patterns associated with dysphoric mood. Psychosom. Med. 71, 580-587. doi: 10.1097/PSY.0b013e3181a2515c

Morency, L.-P., Stratou, G., DeVault, D., Hartholt, A., Lhommet, M., Lucas, G., et al. (2015). "Simsensei demonstration: a perceptive virtual human interviewer for healthcare applications," in Proceedings of the Twenty-Ninth AAAI Conference on Artificial Intelligence, AAAI'15 (Austin, TX: AAAI Press), 4307-4308.

Nasir, M., Jati, A., Shivakumar, P. G., Chakravarthula, S. N., and Georgiou, P. (2016). "Multimodal and multiresolution depression detection from speech and facial landmark features," in Proceedings of the 6th International Workshop on Audio/Visual Emotion Challenge, AVEC '16 (New York, NY: Association for Computing Machinery), 43-50.

National Collaborating Centre for Mental Health (2010). Depression: The Treatment and Management of Depression in Adults. British Psychological Society.

Nesse, and Randolph, M. (2000). Is depression an adaptation? Arch. Gen. Psychiatry 57, 14-20. doi: 10.1001/archpsyc.57.1.14

Ng, A. Y., and Jordan, M. I. (2002). "On discriminative vs. generative classifiers: a comparison of logistic regression and naive bayes," in 15th Annual Conference on Neural Information Processing Systems (NIPS), Vol. 14 (Vancouver, QC), 841-848.

Nicholas, C., Stefan, S., Jarek, K., Sebastian, S., Julien, E., and Thomas F, Q. (2015). A review of depression and suicide risk assessment using speech analysis. Speech Commun. 71, 10-49. doi: 10.1016/j.specom.2015.03.004

Ooi, K. E. B., Lech, M., and Allen, N. B. (2013). Multichannel weighted speech classification system for prediction of major depression in adolescents. IEEE Trans. Biomed. Eng. 60, 497-506. doi: 10.1109/TBME.2012.2228646

Pampouchidou, A., Marias, K., Tsiknakis, M., Simos, P., Yang, F., Lematre, G., et al. (2016a). "Video-based depression detection using local curvelet binary patterns in pairwise orthogonal planes," in 2016 38th Annual International Conference of the IEEE Engineering in Medicine and Biology Society (EMBC) (Orlando), 3835-3838.

Pampouchidou, A., Marias, K., Tsiknakis, M., Simos, P., Yang, F., and Meriaudeau, F. (2015). "Designing a framework for assisting depression severity assessment from facial image analysis," in 2015 IEEE International Conference on Signal and Image Processing Applications (ICSIPA) (Kuala Lumpur), 578-583.

Pampouchidou, A., Simantiraki, O., Fazlollahi, A., Pediaditis, M., Manousos, D., Roniotis, A., et al. (2016b). "Depression assessment by fusing high and low level features from audio, video, and text," in Proceedings of the 6th International Workshop on Audio/Visual Emotion Challenge, AVEC '16 (New York, NY: Association for Computing Machinery), 27-34.

Pampouchidou, A., Simos, P. G., Marias, K., Meriaudeau, F., Yang, F., Pediaditis, M., et al. (2019). Automatic assessment of depression based on visual cues: a systematic review. IEEE Trans. Affect. Comput. 10, 445-470. doi: 10.1109/TAFFC.2017.2724035

Reddy, M. S. (2012). Depression - the global crisis. Indian J. Psychol. Med. 34, 201-203. doi: 10.4103/0253-7176.106011

Rottenberg, J. (2005). Mood and emotion in major depression. Curr. Dir. Psychol. Sci. 14, 167-170. doi: 10.1111/j.0963-7214.2005.00354.x
Rottenberg, J., Gross, J. J., and Gotlib, L. H. (2005). Emotion context insensitivity in major depressive disorder. J. Abnorm. Psychol. 114:627. doi: 10.1037/0021-843X.114.4.627

Schwartz, G. E., Fair, P. L., Salt, P., and Klerman, G. L. (1976). Facial expression and imagery in depression: an electromyographic study. Psychosom. Med. 38, 337-347. doi: 10.1097/00006842-197609000-00006

Sidorov, M., and Minker, W. (2014). "Emotion recognition and depression diagnosis by acoustic and visual features: a multimodal approach," in Proceedings of the 4th International Workshop on Audio/Visual Emotion Challenge, AVEC '14 (New York, NY: Association for Computing Machinery), $81-86$.

Stratou, G., Scherer, S., Gratch, J., and Morency, L. P. (2014). Automatic nonverbal behavior indicators of depression and ptsd: the effect of gender. J. Multimodal User Interfaces 9, 17-29. doi: 10.1007/s12193-014-0161-4

Wang, P., Barrett, F., Martin, E., Milonova, M., Gur, R. E., Gur, R. C., et al. (2008). Automated video-based facial expression analysis of neuropsychiatric disorders. J. Neurosci. Methods 168, 224-238. doi: 10.1016/j.jneumeth.2007.09.030

Wen, L., Li, X., Guo, G., and Zhu, Y. (2015). Automated depression diagnosis based on facial dynamic analysis and sparse coding. IEEE Trans. Inform. Forens. Secur. 10, 1432-1441. doi: 10.1109/TIFS.2015.2414392

Williamson, J. R., Quatieri, T. F., Helfer, B. S., Ciccarelli, G., and Mehta, D. D. (2014). "Vocal and facial biomarkers of depression based on motor incoordination and timing," in Proceedings of the 4th International Workshop on Audio/Visual Emotion Challenge, AVEC '14 (New York, NY: Association for Computing Machinery), 65-72.

Winograd-Gurvich, C., Georgiou-Karistianis, N., Fitzgerald, P., Millist, L., and White, O. (2006). Ocular motor differences between melancholic and non-melancholic depression. J. Affect. Disord. 93, 193-203. doi: $10.1016 /$ j.jad.2006.03.018

Yang, Y., Fairbairn, C., and Cohn, J. F. (2013). Detecting depression severity from vocal prosody. IEEE Trans. Affect. Comput. 4, 142-150. doi: 10.1109/T-AFFC.2012.38

Zhao, X., Huang, D., Dellandrea, E., and Chen, L. (2010). "Automatic 3d facial expression recognition based on a bayesian belief net and a statistical facial feature model," in Proceedings of the 2010 20th International Conference on Pattern Recognition, ICPR '10 (Istanbul: IEEE Computer Society), 3724-3727.

Zhou, X., Jin, K., Shang, Y., and Guo, G. (2020). Visually interpretable representation learning for depression recognition from facial images. IEEE Trans. Affect. Comput. 11, 542-552. doi: 10.1109/TAFFC.2018.28 28819

Conflict of Interest: The authors declare that the research was conducted in the absence of any commercial or financial relationships that could be construed as a potential conflict of interest.

Copyright (๑) 2021 Guo, Yang, Liu, Xu and Hu. This is an open-access article distributed under the terms of the Creative Commons Attribution License (CC BY). The use, distribution or reproduction in other forums is permitted, provided the original author(s) and the copyright owner(s) are credited and that the original publication in this journal is cited, in accordance with accepted academic practice. No use, distribution or reproduction is permitted which does not comply with these terms. 